\title{
Distribution and ECo-Toxicological Risk Assessment of Legacy Persistent Organic Pollutants in Surface Water of Talar, Babolrood and Haraz Rivers
}

\author{
Reza Dahmardeh Behrooz ${ }^{1, *}$, Abbas Esmaili-Sari ${ }^{2}$ and Paromita Chakraborty ${ }^{3}$ \\ 1 Department of Environmental Sciences, Faculty of Natural Resources, University of Zabol, \\ Sistan P.O. Box 98615-538, Iran \\ 2 Department of Environmental Sciences, Faculty of Natural Resources and Marine Sciences, \\ Tarbiat Modares University, Nour, Mazandaran P.O. Box 46414-356, Iran; esmaili@modares.ac.ir \\ 3 Department of Civil Engineering \& SRM Research Institute, SRM Institute of Science and Technology, \\ Kancheepuram district, Tamil Nadu 603203, India; paromitc@srmist.edu.in \\ * Correspondence: dahmardeh_behrooz@yahoo.com or dahmardehbehrooz@uoz.ac.ir; \\ Tel./Fax: +98-054-3323-2600
}

Received: 27 September 2020; Accepted: 30 October 2020; Published: 4 November 2020

check for updates

\begin{abstract}
In the outer Alborz Range in the Northern Province of Iran, the Haraz, Talar and Babolrood Rivers are the three largest rivers where most of the agricultural, aquacultural and industrial activities are found. Total indicator polychlorinated biphenyls (iPCBs) ranged ND-474, ND-273 and ND-559 ng/L in Haraz, Talar and Babolrood Rivers, respectively. Significantly lower $(p<0.05)$ concentrations of DDT, lindane and dieldrin were observed in the upstream stations than downstream stations of both Babolrood and Haraz Rivers. However, in Talar River, PCB residues in upstream was significantly higher $(p<0.05)$ than downstream. Furthermore, PCB-28 in Talar River and PCB-101 and PCB-153 in Bablrood and Haraz Rivers were high in water samples at all stations. A significant difference was observed among the stations. In October, November and December, there was negligible variations in total PCB concentration among the stations. Alarming levels of some of the legacy persistent organic pollutants (POPs) may pose adverse effects on the aquatic species dwelling in the Talar, Babolrood and Haraz Rivers of Iran.
\end{abstract}

Keywords: polychlorinated biphenyls; organochlorine pesticides; rivers; toxicological effects; Iran

\section{Introduction}

Widespread use of chemicals and industrial development during the twentieth century are the major sources for many chemical pollutants to enter the environment [1]. Owing to the ubiquity, toxicity and competency to bio-accumulate and bio-magnify, elucidation of dominant persistent organic pollutants (POPs), viz. polychlorinated biphenyls (PCBs) and organochlorine pesticides (OCPs), are of utmost importance [2,3].

Chemical inertness, insulation properties, oxidative resistance and thermal stability of PCBs have paved the way for their pervasive use in transformers, hydraulic fluids, oils, plastics, adhesives and sealant [4-6]. OCPs were extensively used globally for agricultural activities, pest control, pharmaceutical and healthcare products and control of vector borne diseases. The elevated usages were primarily associated with their lower cost, high availability and remarkable brisk effect on various pests [7-12]. Owing to their adverse detrimental effect on environment and humans, ecological exposure to POPs has become an eminent concern around the globe [7,13-20]

Riverine systems are the principle source of fresh water, sustaining a major proportion of diverse aquatic flora and fauna. Agricultural run-off and industrial and port activities are the major sources for 
OCPs and PCBs in perennial rivers [7]. Rivers and aquatic environments are threatened by agricultural and industrial pollution and should be protected sensitively from such anthropogenic activities [21-25] Contamination with such organic pollutants in the riverine environment has resulted in toxic impact on the biota [2]. River bodies have specific needs for freshwater resources management, and the extent of POP pollution in a riverine ecosystem can be gauged by the assessment of quality of surface water.

As per the national implementation plan of The Islamic Republic of Iran, there are no records of production or usage of pesticidal POPs, and all consumption is associated with import of foreign origin. After the ban on OCPs due to their associated risk, OCP stockpiles of $71,912 \mathrm{~kg}$ containing aldrin, dieldrin, endrin, DDT and HCB were recorded in the warehouse of the Ministry of Jihad-e-Agriculture. The reports depict stockpiles of DDT of $17,800 \mathrm{~kg}$ stored by the Ministry of Health and Medical Education of Iran as part of Malaria Vector Control Program. Usage of PCBs in Iran was reported in sectors including the power and energy, industry and mining, oil, defense and private sectors. Total stockpiles of $8350,000 \mathrm{Kg}$ tons of PCBs including technical grade PCBs, PCBs polluted oil, PCBs polluted equipment, PCBs in current use and equipment containing PCBs have been accounted [26]. In Iran, pronounced ecological change in the riverine ecosystem has been observed due to various anthropogenic activities [27]. During 2000-2004, 101 different categories of pesticides totaling over $27 \times 10^{3}$ tons were used [28]. Seasonal and spatial variation along with point/non-point source identification of various pollutants are the preliminary steps for preservation and purification of any riverine ecosystem [29,30]. Talar, Haraz and Babolrood Rivers have a very important ecological importance in this region and transfer the most pollution from the south to the Caspian Sea [31]. Studies have been conducted on the amount of heavy metal, agricultural pesticides and the impact of municipal wastewater on water quality of these rivers in time periods [27,32-38]. However, this is the first time in Iran that compounds such as PCBs were studied in the entire length of these rivers in two seasons, viz. agricultural cultivation period and rainy season. The major objectives in this study were: (i) to investigate the spatial distribution of selected legacy POPs in the surface water of three major rivers, viz. Talar, Haraz and Babolrood, in northern Iran; (ii) to measure contamination level of 14 legacy POPs (pesticides having ongoing usage; lindane; aldrin; banned organochlorine pesticides including isomers and metabolites of DDTs; and indicator PCB congeners, viz. CB28, CB52, CB101, CB138, CB153 and CB180) using contamination indices; (iii) to estimate the relationship between POPs (PCBs and OCPs) and their possible sources; (iv) to record the monthly variation of the legacy POPs; and (v) to assess the ecotoxicological risk due to POPs contamination and water quality, thereby providing a baseline data for future research.

\section{Material and Methods}

\subsection{Locations of Sampling Stations and Selection of POPS}

This study was conducted along the Haraz, Talar and Babolrood Rivers in north of Iran (Figure 1).

Haraz River originates from Alborz Mountains. The maximum and minimum Elevations heights of the river are 3900 and $20 \mathrm{~m}$, with a slope percentage of $10 \%$. The width of the river varies between 50 and $500 \mathrm{~m}$. Haraz River has a length of $185 \mathrm{~km}$ and a catchment area of $60 \mathrm{Km}^{2}$. The amount of precipitation is $832 \mathrm{~mm} /$ year with a discharge of $30.18 \mathrm{~m}^{3} / \mathrm{s}$ (in 2006) [34,39]. The river flows into the Caspian Sea after passing through many villages, as well as the cities of Amol and Sorkhrud.

The Talar River has a variable width of 30-60 m and in about $150 \mathrm{~km}$ long. It covers an area of about $2845 \mathrm{~km}^{2}$ in the Caspian Sea basin, and the slope of Talar River is about $7 \%$. The headwater of this river is the Alborz Mountains, which are located $3850 \mathrm{~m}$ above sea level [40]. The river passes through many villages and the vicinity of Ghaemshahr city with a population of 320,741. The annual precipitation in Ghaemshahr region between 1951 and 2013 was $729.8 \mathrm{~mm}$.

Babolrood River has a variable width of 40-60 $\mathrm{m}$ and in $110.7 \mathrm{~km}$ long. It has maximum and minimum Elevations heights of 3180 and $10 \mathrm{~m}$, respectively. The steepest slope of the river is $0.74 \%$ and its catchment area is $1746.42 \mathrm{~km}^{2}$. The slope of Baboolrood River is about $7 \%$, and its annual mean discharge varies from 3.44 to $16.67 \mathrm{~m}^{3} / \mathrm{s}$. Haraz River has the most restaurants and industrial centers 
that dump their sewage into this river, followed by aquaculture and agriculture. However, Talar and Babolrood Rivers have the most agricultural use, followed by industrial and then aquaculture discharge.

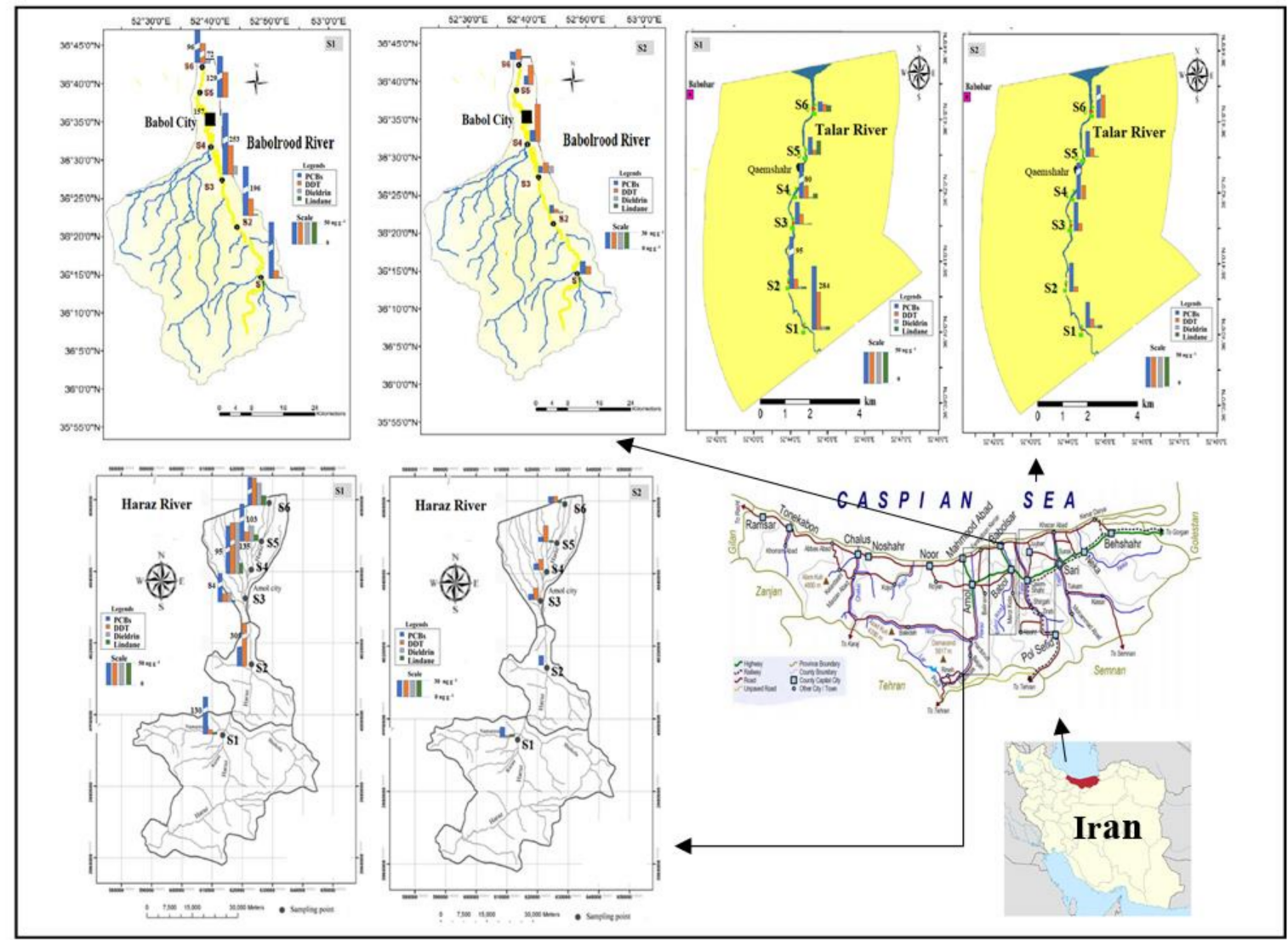

Figure 1. Sampling sites on the Talar, Haraz and Babolrood Rivers and distribution of $\sum_{7} \mathrm{PCBs}$, DDT, dieldrine and lindane. S1-S6, Stations 1-6 on each river.

\subsection{Collection of Samples}

To determine the sampling points along the river, we considered discharge points of industrial wastes, fish farming, agricultural runoff and other sources of pollutants into the river. The qualitative effective branches of river water and reachability of the sites were also considered, and, accordingly, six sampling stations (S1-S6) (Figure 1) were selected along each river route to cover the entire length of the river and pollution factors along the river route. Sampling of river water was done every two weeks for six months (July-December), including the summer season (July-September), which is the agricultural season with the use of pesticides as well as the season with reduced rainfall in the region, and the autumn season (October-December), which is the rainy season in the region with increased river waters and reduced agriculture activity.

High-grade amber glass bottles ( $1 \mathrm{~L})$ were used for collecting surface water samples. In total, 204 samples (68 samples per river: each station was sampled 12 times during 180 days of study, i.e., one sample from each station every 15 days) were analyzed for 14 POPs during the two-season study period (from July to December 2008) (Figure 1). Before sample collection, the glass jars were cleaned with distilled water. The bottles were rinsed using acetone and hexane in sequence to remove traces of organic contaminants. Water samples were collected completely in each glass jar to avoid air bubbles in the glass. Samples were transferred to the laboratory and filtered $(0.45-\mu \mathrm{m}$ fiber glass filters) to get rid of particulate matter and then stored at $4{ }^{\circ} \mathrm{C}$ prior to analysis. Solid-phase extraction (Supelco) was used following established procedures for extracted river water samples [41] with minor modifications. The cartridges were conditioned with methanol $(2 \times 5 \mathrm{~mL})$ followed by deionized 
water $(2 \times 5 \mathrm{~mL})$. Internal standards $(\varepsilon-\mathrm{HCH}$ and PCB 143) were added to $1000-\mathrm{mL}$ water samples (spiked) and then passed under vacuum $\left(7 \mathrm{~mL} \mathrm{~min}^{-1}\right)$ through the cartridges. The cartridges were eluted using $15 \mathrm{~mL}$ of dichloromethane and cleaned with $\mathrm{Na}_{2} \mathrm{SO}_{4}$ (anhydrous) for removal of water from the extracts. Finally, the extracts were concentrated under a gentle stream of nitrogen.

\subsection{Extraction and Analysis}

Chemicals were procured from Merck Inc. (Darmastadt, Germany). Standards for indicator PCBs (IUPAC Nos. 28, 52, 101, 138, 153, 143 and 180) and OCPs (lindane $\left(\gamma\right.$-HCH), $\varepsilon-H C H, 2,4^{\prime}-D D E$, 4,4'-DDE, 2,4' -DDT, 4,4' -DDT and 4,4'-DDD) were obtained from Dr. Ehrenstorfer Laboratories (Augsburg, Germany). Gas chromatograph (Danni 1000 GC-ECD, PTV injector Monza, Italy) was used for quantification of POPs with a fused silica capillary column (Optima 5), of dimension $60 \mathrm{~m} \times 0.25 \mathrm{~mm}$. A fused silica capillary column with $0.5-\mu \mathrm{m}$ film thickness was also used. Clarity Chromatography Software (Data, Apex Prague, Czech Republic) was used to analyze the surface under peaks. Helium was used as the carrier gas with high-purity (99.999\%) and flow rate of $2 \mathrm{~mL} / \mathrm{min}$. The operating conditions were: after $30 \mathrm{~s}$ split open; temperature program of hold for $1 \mathrm{~min}$ at $100{ }^{\circ} \mathrm{C}$, drop the temperature at $10^{\circ} \mathrm{C} / \mathrm{min}$ to $240{ }^{\circ} \mathrm{C}$ and hold for $1 \mathrm{~min}$, drop at $1^{\circ} \mathrm{C} / \mathrm{min}$ to $260{ }^{\circ} \mathrm{C}$ and hold $1 \mathrm{~min}$ and finally drop at $10^{\circ} \mathrm{C} / \mathrm{min}$ to $300{ }^{\circ} \mathrm{C}$ and hold $10 \mathrm{~min}$; the temperatures in PTV injection port and detector were 250 and $300{ }^{\circ} \mathrm{C}$, respectively; and injection volume was $1 \mu \mathrm{L}$. The POP residues were quantitatively determined by comparing the area under each peak with the area under the internal standard peak. Correlation coefficients $\left(R^{2}>0.998\right)$ were plotted with daily external standard multi point calibration curves using peak areas versus injected concentrations. For each analysis, a standard deviation of signal was obtained, and the mean standard deviation was determined.

\subsection{Quality Assurance and Quality Control}

A seven-point calibration curve for all contaminants within 5-500 ng/L was used for quantification. Recoveries for OCPs and PCBs were $80-115 \%$ and $90-118 \%$, respectively. The procedural blanks were run every fifteen samples to monitor cross-contamination. LOQ (S/N = 10:1) for OCPs and PCBs varied 0.6-3 and 0.5-2 ng/L respectively. All method and procedural blanks were below the LOQ of all the quantified analytes. Intra- and inter-day variability was analyzed to evaluate the repeatability and precision of the analytical method. The relative standard deviation (RSD) based on repeatability was obtained in the ranges of $8-15 \%$ and $7-12 \%$ for OCPs and PCBs for intra- and inter-day, respectively.

\subsection{Statistical Analysis}

The non-detectable concentrations were substituted with zero while calculating the mean concentrations of OCPs and PCBs in surface water samples from rivers and for statistical purposes $(<\mathrm{LOQ}=0$, i.e., lower bound, LB). The data distribution was tested using Kolmogorov-Smirnov test. These data were not normal and became normal after log-transformation. The one-way analysis of variance (ANOVA) was used for statistical analysis of the dataset. The Tukey multiple-comparison test was applied to ANOVA to determine the statistical differences among the stations. POPs concentration with respect to season was subjected to an independent $t$ test. For statistical analysis, the SPSS Software (Version 16.5) was utilized and level of significance was set in $p<0.05$.

\subsection{Ecotoxicological Risk}

Ecotoxicological risk was estimated for DDT and lindane using hazard quotient (HQ) approach with the formula:

$$
\mathrm{HQ}=\mathrm{MEC} / \mathrm{PNEC}
$$

where MEC is the measured environmental concentration in surface water of the three rivers and PNEC is the predicted no-effect concentration for various species in the aqueous environment given elsewhere [7]. 


\section{Results and Discussion}

\subsection{Levels of Selected Legacy POPs and Comparison with Other Studies}

The OCP residues quantified from Talar, Babolrood and Haraz Rivers are given in Tables 1-3. The concentrations of DDT, dieldrin and lindane in Haraz River were significantly different $(p<0.05)$ from other rivers. Interestingly, around this river there is intensive agricultural and aquaculture activity. It has been observed that water bodies near such intensive agricultural areas tend to be contaminated due to the application of agricultural pesticides $[42,43]$. Among the three detected pesticides DDT, lindane and dieldrin, the mean concentration of DDT was significantly $(p<0.05)$ higher than lindane and dieldrin (Tables 1-3). The mean concentrations of DDTs, lindane and dieldrin are shown in Tables 1-3.

Table 1. Concentrations (ng/L) (mean and range) of POPs in water samples from Stations 1-6 of Talar River collected from Iran. LOQ, limit of quantification; S1-S6, Stations 1-6 in each river.

\begin{tabular}{|c|c|c|c|c|c|c|c|}
\hline & S1 & S2 & S3 & S4 & S5 & S6 & Total \\
\hline & $\begin{array}{l}\text { Mean } \\
\text { (Range) }\end{array}$ & $\begin{array}{l}\text { Mean } \\
\text { (Range) }\end{array}$ & $\begin{array}{l}\text { Mean } \\
\text { (Range) }\end{array}$ & $\begin{array}{l}\text { Mean } \\
\text { (Range) }\end{array}$ & $\begin{array}{l}\text { Mean } \\
\text { (Range) }\end{array}$ & $\begin{array}{l}\text { Mean } \\
\text { (Range) }\end{array}$ & $\begin{array}{l}\text { Mean } \\
\text { (Range) }\end{array}$ \\
\hline $\mathrm{PCB}_{28}$ & $\begin{array}{c}21 \\
(<\text { LOQ- } 84)\end{array}$ & $\begin{array}{c}29 \\
(<\text { LOQ-123) }\end{array}$ & $\begin{array}{c}23 \\
(<\mathrm{LOQ}-202)\end{array}$ & $\begin{array}{c}33 \\
(<\mathrm{LOQ}-143)\end{array}$ & $\begin{array}{c}21 \\
(<\text { LOQ-109) }\end{array}$ & $\begin{array}{c}16 \\
(<\text { LOQ-63) }\end{array}$ & $\begin{array}{c}24 \\
(<\text { LOQ-202) }\end{array}$ \\
\hline $\mathrm{PCB}_{52}$ & $\begin{array}{c}16 \\
(<\text { LOQ-107) }\end{array}$ & $\begin{array}{c}10 \\
(<\text { LOQ }-83)\end{array}$ & 7 & $\begin{array}{c}8 \\
(<\text { LOQ-58) }\end{array}$ & $\begin{array}{c}7 \\
(<\text { LOQ-27) }\end{array}$ & $26(<$ LOQ-148) & $\begin{array}{c}12 \\
(<\text { LOQ }-148)\end{array}$ \\
\hline $\mathrm{PCB}_{101}$ & $\begin{array}{c}19 \\
(0-70)\end{array}$ & $\begin{array}{c}2 \\
(<\text { LOQ }-10)\end{array}$ & $<$ LOQ & $\begin{array}{c}9 \\
(<\text { LOQ-93) }\end{array}$ & $\begin{array}{c}1 \\
(<\text { LOQ }-10)\end{array}$ & $(<\mathrm{LOQ})$ & $\begin{array}{c}5 \\
(<\text { LOQ-93) }\end{array}$ \\
\hline $\mathrm{PCB}_{138}$ & $\begin{array}{c}21 \\
(<\text { LOQ-236) }\end{array}$ & 0 & $<\mathrm{LOQ}$ & $\begin{array}{c}4 \\
(<\mathrm{LOQ}-46)\end{array}$ & $(<\mathrm{LOQ})$ & $(<\mathrm{LOQ})$ & $\begin{array}{c}5 \\
(<\text { LOQ-236) }\end{array}$ \\
\hline $\mathrm{PCB}_{153}$ & $\begin{array}{c}44 \\
(<\mathrm{LOQ}-284)\end{array}$ & $\begin{array}{c}23 \\
(<\mathrm{LOQ}-146)\end{array}$ & $\begin{array}{c}9 \\
(<\mathrm{LOQ}-89)\end{array}$ & $\begin{array}{c}12 \\
(<\mathrm{LOQ}-54)\end{array}$ & $\begin{array}{c}5 \\
(<\mathrm{LOQ}-44)\end{array}$ & $\begin{array}{c}2 \\
(<\mathrm{LOQ}-12)\end{array}$ & $\begin{array}{c}16 \\
(<\mathrm{LOQ}-248)\end{array}$ \\
\hline $\mathrm{PCB}_{180}$ & $\begin{array}{c}33 \\
(<\mathrm{LOQ}-237)\end{array}$ & $\begin{array}{c}6 \\
(<\mathrm{LOQ}-27)\end{array}$ & $\begin{array}{c}4 \\
(<\mathrm{LOQ}-23)\end{array}$ & $\begin{array}{c}15 \\
(<\text { LOQ-95) }\end{array}$ & $\begin{array}{c}3 \\
(<\mathrm{LOQ}-15)\end{array}$ & $\begin{array}{c}3 \\
(<\text { LOQ-12) }\end{array}$ & $\begin{array}{c}11 \\
(<\text { LOQ-237) }\end{array}$ \\
\hline$\sum_{7} \mathrm{PCBs}$ & $\begin{array}{c}78 \\
(<\text { LOQ-273) }\end{array}$ & $(<$ LOQ-174) & $\begin{array}{c}43 \\
(<\text { LOQ-215) }\end{array}$ & $\begin{array}{c}81 \\
(\mathrm{LOQ}-261)\end{array}$ & $(2-119)$ & $\begin{array}{c}46 \\
(<\text { LOQ-119) }\end{array}$ & $\begin{array}{c}59 \\
(<\text { LOQ-273) }\end{array}$ \\
\hline $4,4^{\prime}-\mathrm{DDT}$ & $\begin{array}{c}19 \\
(<\text { LOQ-139) }\end{array}$ & $\begin{array}{c}5 \\
(<\text { LOQ }-131)\end{array}$ & $\begin{array}{c}5 \\
(<\text { LOQ }-48)\end{array}$ & $\begin{array}{c}6 \\
\text { (LOQ-60) }\end{array}$ & $\begin{array}{c}7 \\
(<\mathrm{LOQ}-32)\end{array}$ & $\begin{array}{c}14 \\
(<\text { LOQ-101) }\end{array}$ & $\begin{array}{c}9 \\
(<\text { LOQ-139) }\end{array}$ \\
\hline $2,4^{\prime}-\mathrm{DDT}$ & $\begin{array}{c}14 \\
(<\text { LOQ-104) }\end{array}$ & $\begin{array}{c}4 \\
(<\text { LOQ-16) }\end{array}$ & $\begin{array}{c}5 \\
(<\mathrm{LOQ}-21)\end{array}$ & $\begin{array}{c}13 \\
(\text { LOQ-123) }\end{array}$ & $\begin{array}{c}3 \\
(<\mathrm{LOQ}-25)\end{array}$ & $\begin{array}{c}11 \\
(<\text { LOQ-75) }\end{array}$ & $\begin{array}{c}8 \\
(<\text { LOQ }-123)\end{array}$ \\
\hline $4,4^{\prime}-\mathrm{DDE}$ & $<$ LOQ & 0 & $<$ LOQ & $(<\mathrm{LOQ})$ & $(<$ LOQ $)$ & $(<\mathrm{LOQ})$ & $(<$ LOQ $)$ \\
\hline $2,4^{\prime}-\mathrm{DDE}$ & $\begin{array}{c}2 \\
(<\text { LOQ }-10)\end{array}$ & $\begin{array}{c}1 \\
(<\mathrm{LOQ}-10)\end{array}$ & $\begin{array}{c}3 \\
(<\mathrm{LOQ}-10)\end{array}$ & $\begin{array}{c}2 \\
(<\text { LOQ }-10)\end{array}$ & $\begin{array}{c}1 \\
(<\mathrm{LOQ}-4)\end{array}$ & $\begin{array}{c}1 \\
(<\text { LOQ }-10)\end{array}$ & $\begin{array}{c}2 \\
(<\mathrm{LOQ}-10)\end{array}$ \\
\hline $4,4^{\prime}-\mathrm{DDD}$ & $\begin{array}{c}3 \\
(<\mathrm{LOQ}-7)\end{array}$ & $(<\mathrm{LOQ}-5)$ & $\begin{array}{c}1 \\
(<\mathrm{LOQ}-5)\end{array}$ & $\begin{array}{c}1 \\
(<\mathrm{LOQ}-5)\end{array}$ & $(<$ LOQ -2$)$ & $(<\mathrm{LOQ})$ & $\begin{array}{c}1 \\
(<\mathrm{LOQ}-7)\end{array}$ \\
\hline$\sum$ DDTS & $\begin{array}{c}38 \\
(<\mathrm{LOQ}-260)\end{array}$ & $\begin{array}{c}11 \\
(<\text { LOQ-33) }\end{array}$ & $\begin{array}{c}13 \\
(<\text { LOQ }-49)\end{array}$ & $\begin{array}{c}22 \\
(<\text { LOQ-123) }\end{array}$ & $\begin{array}{c}11 \\
(<\text { LOQ }-32)\end{array}$ & $\begin{array}{c}26 \\
(<\text { LOQ-101) }\end{array}$ & $\begin{array}{c}21 \\
(<\text { LOQ-260) }\end{array}$ \\
\hline Dieldrin & $\begin{array}{c}2 \\
(<\text { LOQ-6) }\end{array}$ & $\begin{array}{c}1 \\
(<\text { LOQ }-3)\end{array}$ & $\begin{array}{c}1 \\
(<\text { LOQ }-4)\end{array}$ & $\begin{array}{c}<\text { LOQ } \\
(<\text { LOQ-3) }\end{array}$ & $(<\mathrm{LOQ})$ & $(<\mathrm{LOQ})$ & $\begin{array}{c}1 \\
(<\text { LOQ }-6)\end{array}$ \\
\hline Lindane & $\begin{array}{c}3 \\
(<\text { LOQ-6) }\end{array}$ & $\begin{array}{c}1 \\
(<\mathrm{LOQ}-5)\end{array}$ & $<$ LOQ & $\begin{array}{c}3 \\
(<\text { LOQ }-10)\end{array}$ & $\begin{array}{c}10 \\
(<\text { LOQ-90) }\end{array}$ & $\begin{array}{c}4 \\
(<\text { LOQ-30) }\end{array}$ & $\begin{array}{c}3 \\
(<\mathrm{LOQ}-90)\end{array}$ \\
\hline
\end{tabular}

Table 2. Concentrations (ng/L) (mean and range) of POPs in water samples of Babolrood River collected from Iran. LOQ, limit of quantification.

\begin{tabular}{|c|c|c|c|c|c|c|c|}
\hline & S1 & S2 & S3 & S4 & S5 & S6 & Total \\
\hline Babolrood & $\begin{array}{c}\text { Mean } \\
\text { (Range) }\end{array}$ & $\begin{array}{c}\text { Mean } \\
\text { (Range) }\end{array}$ & $\begin{array}{c}\text { Mean } \\
\text { (Range) }\end{array}$ & $\begin{array}{c}\text { Mean } \\
\text { (Range) }\end{array}$ & $\begin{array}{c}\text { Mean } \\
\text { (Range) }\end{array}$ & $\begin{array}{c}\text { Mean } \\
\text { (Range) }\end{array}$ & $\begin{array}{c}\text { Mean } \\
\text { (Range) }\end{array}$ \\
\hline $\mathrm{PCB}_{28}$ & $\begin{array}{c}12 \\
(<\mathrm{LOQ}-61)\end{array}$ & $\begin{array}{c}5 \\
(<\mathrm{LOQ}-30)\end{array}$ & $\begin{array}{c}1 \\
(<\text { LOQ }-15)\end{array}$ & $\begin{array}{c}3 \\
(<\mathrm{LOQ}-23)\end{array}$ & $\begin{array}{c}6 \\
(<\mathrm{LOQ}-37)\end{array}$ & $\begin{array}{c}3 \\
(<\mathrm{LOQ}-20)\end{array}$ & $\begin{array}{c}5 \\
(<\text { LOQ-61) }\end{array}$ \\
\hline $\mathrm{PCB}_{52}$ & $(<\mathrm{LOQ}-24)$ & $\begin{array}{c}9 \\
(<\mathrm{LOQ}-20)\end{array}$ & $\begin{array}{c}10 \\
(<\mathrm{LOQ}-52)\end{array}$ & $\begin{array}{c}16 \\
(<\text { LOQ-55) }\end{array}$ & $\begin{array}{c}15 \\
(<\mathrm{LOQ}-58)\end{array}$ & $(<\mathrm{LOQ}-34)$ & $(<\mathrm{LOQ}-58)$ \\
\hline $\mathrm{PCB}_{101}$ & $\begin{array}{c}65 \\
(<\mathrm{LOQ}-457)\end{array}$ & $\begin{array}{c}53 \\
(<\text { LOQ }-431)\end{array}$ & $\begin{array}{c}108 \\
(<\text { LOQ-550) }\end{array}$ & $\begin{array}{c}22 \\
(<\mathrm{LOQ}-146)\end{array}$ & $\begin{array}{c}5 \\
(<\mathrm{LOQ}-44)\end{array}$ & $\begin{array}{c}9 \\
(<\mathrm{LOQ}-64)\end{array}$ & $\begin{array}{c}44 \\
(<\text { LOQ-550) }\end{array}$ \\
\hline $\mathrm{PCB}_{138}$ & $(<\mathrm{LOQ})$ & $\begin{array}{c}12 \\
(<\text { LOQ }-107)\end{array}$ & $(<\mathrm{LOQ})$ & $\begin{array}{c}10 \\
(<\text { LOQ-114) }\end{array}$ & $\begin{array}{c}10 \\
(<\mathrm{LOQ}-111)\end{array}$ & $(<\mathrm{LOQ})$ & $\begin{array}{c}5 \\
(<\text { LOQ }-114)\end{array}$ \\
\hline
\end{tabular}


Table 2. Cont

\begin{tabular}{|c|c|c|c|c|c|c|c|}
\hline & S1 & S2 & S3 & S4 & S5 & S6 & Total \\
\hline Babolrood & $\begin{array}{c}\text { Mean } \\
\text { (Range) }\end{array}$ & $\begin{array}{l}\text { Mean } \\
\text { (Range) }\end{array}$ & $\begin{array}{l}\text { Mean } \\
\text { (Range) }\end{array}$ & $\begin{array}{l}\text { Mean } \\
\text { (Range) }\end{array}$ & $\begin{array}{l}\text { Mean } \\
\text { (Range) }\end{array}$ & $\begin{array}{l}\text { Mean } \\
\text { (Range) }\end{array}$ & $\begin{array}{l}\text { Mean } \\
\text { (Range) }\end{array}$ \\
\hline $\mathrm{PCB}_{153}$ & $\begin{array}{c}18 \\
(<\mathrm{LOQ}-123)\end{array}$ & $\begin{array}{c}10 \\
(<\mathrm{LOQ}-103)\end{array}$ & $\begin{array}{c}2 \\
(<\mathrm{LOQ}-18)\end{array}$ & $\begin{array}{c}16 \\
(<\mathrm{LOQ}-114)\end{array}$ & $\begin{array}{c}22 \\
(<\mathrm{LOQ}-120)\end{array}$ & $\begin{array}{c}23 \\
(<\mathrm{LOQ}-170)\end{array}$ & $\begin{array}{c}15 \\
(<\mathrm{LOQ}-170)\end{array}$ \\
\hline $\mathrm{PCB}_{180}$ & $\begin{array}{c}14 \\
(<\mathrm{LOQ}-81)\end{array}$ & $\begin{array}{c}8 \\
(<\mathrm{LOQ}-78)\end{array}$ & $\begin{array}{c}2 \\
(<\mathrm{LOQ}-16)\end{array}$ & $\begin{array}{c}20 \\
(<\mathrm{LOQ}-143)\end{array}$ & $\begin{array}{c}10 \\
(<\mathrm{LOQ}-86)\end{array}$ & $\begin{array}{c}7 \\
(<\mathrm{LOQ}-54)\end{array}$ & $\begin{array}{c}10 \\
(<\mathrm{LOQ}-143)\end{array}$ \\
\hline$\sum$ PCBs & $\begin{array}{c}116 \\
(1-486)\end{array}$ & $\begin{array}{c}97 \\
(<\mathrm{LOQ}-436)\end{array}$ & $\begin{array}{c}122 \\
(<\text { LOQ-559) }\end{array}$ & $\begin{array}{c}87 \\
(<\mathrm{LOQ}-406)\end{array}$ & $\begin{array}{c}68 \\
(11-341)\end{array}$ & $\begin{array}{c}52 \\
(2-180)\end{array}$ & $\begin{array}{c}91 \\
(<\mathrm{LOQ}-559)\end{array}$ \\
\hline $4,4^{\prime}-\mathrm{DDT}$ & $\begin{array}{c}1 \\
(<\mathrm{LOQ}-9)\end{array}$ & $\begin{array}{c}7 \\
(<\mathrm{LOQ}-44)\end{array}$ & $\begin{array}{c}3 \\
(<\text { LOQ }-19)\end{array}$ & $\begin{array}{c}48 \\
(<\text { LOQ }-256)\end{array}$ & $\begin{array}{c}19 \\
(<\text { LOQ-85) }\end{array}$ & $\begin{array}{c}12 \\
(<\mathrm{LOQ}-43)\end{array}$ & $\begin{array}{c}15 \\
(<\mathrm{LOQ}-256)\end{array}$ \\
\hline $2,4^{\prime}-\mathrm{DDT}$ & $\begin{array}{c}11 \\
(<\mathrm{LOQ}-81)\end{array}$ & $\begin{array}{c}8 \\
(<\mathrm{LOQ}-30)\end{array}$ & $\begin{array}{c}8 \\
(<\mathrm{LOQ}-40)\end{array}$ & $\begin{array}{c}8 \\
(<\text { LOQ-68) }\end{array}$ & $\begin{array}{c}4 \\
(<\mathrm{LOQ}-29)\end{array}$ & $\begin{array}{c}15 \\
(<\mathrm{LOQ}-114)\end{array}$ & $\begin{array}{c}9 \\
(<\mathrm{LOQ}-114)\end{array}$ \\
\hline $4,4^{\prime}-\mathrm{DDE}$ & $(<\mathrm{LOQ}-1)$ & $<\mathrm{LOQ}-$ & $\begin{array}{c}1 \\
(<\mathrm{LOQ}-6)\end{array}$ & $\begin{array}{c}2 \\
(<\mathrm{LOQ}-6)\end{array}$ & $\begin{array}{c}1 \\
(<\mathrm{LOQ}-5)\end{array}$ & $\begin{array}{c}1 \\
(<\mathrm{LOQ}-4)\end{array}$ & $\begin{array}{c}1 \\
(<\mathrm{LOQ}-6)\end{array}$ \\
\hline $2,4^{\prime}-\mathrm{DDE}$ & $(<$ LOQ-2) & $\begin{array}{c}1 \\
(<\mathrm{LOQ}-3)\end{array}$ & $\begin{array}{c}21 \\
(<\mathrm{LOQ}-100)\end{array}$ & $\begin{array}{c}3 \\
(<\mathrm{LOQ}-20)\end{array}$ & $(<\mathrm{LOQ}-10)$ & $\begin{array}{c}5 \\
(<\mathrm{LOQ}-30)\end{array}$ & $\begin{array}{c}5 \\
(<\mathrm{LOQ}-100)\end{array}$ \\
\hline $4,4^{\prime}-\mathrm{DDD}$ & $\begin{array}{c}1 \\
(<\mathrm{LOQ}-5)\end{array}$ & $\begin{array}{c}1 \\
(<\mathrm{LOQ}-5)\end{array}$ & $\begin{array}{c}1 \\
(<\mathrm{LOQ}-4)\end{array}$ & $\begin{array}{c}6 \\
(<\mathrm{LOQ}-30)\end{array}$ & $\begin{array}{c}14 \\
(<\mathrm{LOQ}-100)\end{array}$ & $\begin{array}{c}11 \\
(<\text { LOQ-60) }\end{array}$ & $\begin{array}{c}5 \\
(<\text { LOQ-100) }\end{array}$ \\
\hline$\sum$ DDTS & $\begin{array}{c}14 \\
(<\mathrm{LOQ}-83)\end{array}$ & $\begin{array}{c}17 \\
(<\mathrm{LOQ}-83)\end{array}$ & $(<\mathrm{LOQ}-130)$ & $(<\mathrm{LOQ}-257)$ & $\begin{array}{c}39 \\
(<\mathrm{LOQ}-100)\end{array}$ & $\begin{array}{c}43 \\
(<\mathrm{LOQ}-174)\end{array}$ & $\begin{array}{c}36 \\
(<\mathrm{LOQ}-257)\end{array}$ \\
\hline Dieldrin & $\begin{array}{c}2 \\
(<\mathrm{LOQ}-6)\end{array}$ & $\begin{array}{c}2 \\
(<\text { LOQ-6) }\end{array}$ & $\begin{array}{c}10 \\
(<\mathrm{LOQ}-50)\end{array}$ & $\begin{array}{c}11 \\
(<\mathrm{LOQ}-50)\end{array}$ & $<\mathrm{LOQ}$ & $\begin{array}{c}2 \\
(<\text { LOQ }-20)\end{array}$ & $\begin{array}{c}5 \\
(<\mathrm{LOQ}-50)\end{array}$ \\
\hline Lindane & $(<\mathrm{LOQ})$ & $<\mathrm{LOQ}$ & $<$ LOQ & $<$ LOQ & $<\mathrm{LOQ}$ & $<$ LOQ & $<\mathrm{LOQ}$ \\
\hline
\end{tabular}

Table 3. Concentrations (mean and range) in ng/L of POPs in water samples of Haraz River collected from Iran. LOQ, limit of quantification.

\begin{tabular}{|c|c|c|c|c|c|c|c|}
\hline & S1 & S2 & S3 & S4 & S5 & S6 & Total \\
\hline Haraz & $\begin{array}{c}\text { Mean } \\
\text { (Range) }\end{array}$ & $\begin{array}{c}\text { Mean } \\
\text { (Range) }\end{array}$ & $\begin{array}{c}\text { Mean } \\
\text { (Range) }\end{array}$ & $\begin{array}{c}\text { Mean } \\
\text { (Range) }\end{array}$ & $\begin{array}{c}\text { Mean } \\
\text { (Range) }\end{array}$ & $\begin{array}{c}\text { Mean } \\
\text { (Range) }\end{array}$ & $\begin{array}{c}\text { Mean } \\
\text { (Range) }\end{array}$ \\
\hline $\mathrm{PCB}_{28}$ & $\begin{array}{c}9 \\
(<\mathrm{LOQ}-51)\end{array}$ & $\begin{array}{c}7 \\
(<\mathrm{LOQ}-34)\end{array}$ & $\begin{array}{c}2 \\
(<\mathrm{LOQ}-10)\end{array}$ & $\begin{array}{c}5 \\
(<\mathrm{LOQ}-27)\end{array}$ & $\begin{array}{c}3 \\
(<\mathrm{LOQ}-14)\end{array}$ & $\begin{array}{c}7 \\
(<\mathrm{LOQ}-47)\end{array}$ & $\begin{array}{c}6 \\
(<\mathrm{LOQ}-51)\end{array}$ \\
\hline $\mathrm{PCB}_{52}$ & $\begin{array}{c}15 \\
(<\mathrm{LOQ}-75)\end{array}$ & $\begin{array}{c}7 \\
(<\mathrm{LOQ}-20)\end{array}$ & $\begin{array}{c}5 \\
(<\mathrm{LOQ}-15)\end{array}$ & $\begin{array}{c}6 \\
(<\mathrm{LOQ}-30)\end{array}$ & $\begin{array}{c}11 \\
(<\mathrm{LOQ}-70)\end{array}$ & $\begin{array}{c}14 \\
(<\mathrm{LOQ}-110)\end{array}$ & $\begin{array}{c}10 \\
(<\mathrm{LOQ}-110)\end{array}$ \\
\hline $\mathrm{PCB}_{101}$ & $\begin{array}{c}8 \\
(<\mathrm{LOQ}-59)\end{array}$ & $\begin{array}{c}2 \\
(<\mathrm{LOQ}-12)\end{array}$ & $\begin{array}{c}3 \\
(<\mathrm{LOQ}-24)\end{array}$ & $(<\mathrm{LOQ}-184)$ & $(<\mathrm{LOQ}-170)$ & $(<\mathrm{LOQ}-181)$ & $(<\mathrm{LOQ}-184)$ \\
\hline $\mathrm{PCB}_{138}$ & $\begin{array}{c}5 \\
(<\mathrm{LOQ}-60)\end{array}$ & $(<\mathrm{LOQ}-1)$ & $<$ LOQ & $<\mathrm{LOQ}$ & $<\mathrm{LOQ}$ & & $\begin{array}{c}1 \\
(<\mathrm{LOQ}-60)\end{array}$ \\
\hline $\mathrm{PCB}_{153}$ & $\begin{array}{c}29 \\
(<\mathrm{LOQ}-223)\end{array}$ & $\begin{array}{c}7 \\
(<\mathrm{LOQ}-5)\end{array}$ & $\begin{array}{c}31 \\
(<\mathrm{LOQ}-240)\end{array}$ & $\begin{array}{c}8 \\
(<\mathrm{LOQ}-40)\end{array}$ & $\begin{array}{c}6 \\
(<\mathrm{LOQ}-32)\end{array}$ & $\begin{array}{c}7 \\
(<\mathrm{LOQ}-39)\end{array}$ & $\begin{array}{c}15 \\
(<\mathrm{LOQ}-240)\end{array}$ \\
\hline $\mathrm{PCB}_{180}$ & $\begin{array}{c}9 \\
(<\mathrm{LOQ}-59)\end{array}$ & $\begin{array}{c}4 \\
(<\text { LOQ-29) }\end{array}$ & $\begin{array}{c}3 \\
(<\mathrm{LOQ}-31)\end{array}$ & $\begin{array}{c}6 \\
(<\text { LOQ }-48)\end{array}$ & $\begin{array}{c}4 \\
(<\mathrm{LOQ}-48)\end{array}$ & $\begin{array}{c}5 \\
(<\mathrm{LOQ}-59)\end{array}$ & $\begin{array}{c}5 \\
(<\mathrm{LOQ}-59)\end{array}$ \\
\hline$\sum \mathrm{PCBs}$ & $\begin{array}{c}75 \\
(<\mathrm{LOQ}-474)\end{array}$ & $\begin{array}{c}27 \\
(<\mathrm{LOQ}-70)\end{array}$ & $\begin{array}{c}44 \\
(<\mathrm{LOQ}-240)\end{array}$ & $\begin{array}{c}54 \\
(3-232)\end{array}$ & $\begin{array}{c}52 \\
(1-258)\end{array}$ & $\begin{array}{c}62 \\
(2-279)\end{array}$ & $\begin{array}{c}53 \\
(<\mathrm{LOQ}-474)\end{array}$ \\
\hline $4,4^{\prime}-\mathrm{DDT}$ & $\begin{array}{c}1 \\
(<\mathrm{LOQ}-8)\end{array}$ & $<$ LOQ & $\begin{array}{c}2 \\
(<\mathrm{LOQ}-11)\end{array}$ & $\begin{array}{c}5 \\
(<\mathrm{LOQ}-44)\end{array}$ & $\begin{array}{c}1 \\
(<\mathrm{LOQ}-4)\end{array}$ & $\begin{array}{c}1 \\
(<\mathrm{LOQ}-5)\end{array}$ & $\frac{2}{(<\mathrm{LOQ}-44)}$ \\
\hline $2,4^{\prime}-\mathrm{DDT}$ & $\begin{array}{c}3 \\
(<\mathrm{LOQ}-33)\end{array}$ & $\begin{array}{c}138 \\
(<\mathrm{LOQ}-1490)\end{array}$ & $\begin{array}{c}1 \\
(<\mathrm{LOQ}-10)\end{array}$ & $\begin{array}{c}8 \\
(<\mathrm{LOQ}-80)\end{array}$ & $\begin{array}{c}5 \\
(<\mathrm{LOQ}-50)\end{array}$ & $\begin{array}{c}6 \\
(<\mathrm{LOQ}-50)\end{array}$ & $\begin{array}{c}26 \\
(<\mathrm{LOQ}-1490)\end{array}$ \\
\hline $4,4^{\prime}-\mathrm{DDE}$ & $<\mathrm{LOQ}$ & $<\mathrm{LOQ}$ & $\begin{array}{c}13 \\
(<\mathrm{LOQ}-50)\end{array}$ & $\begin{array}{c}31 \\
(<\mathrm{LOQ}-258)\end{array}$ & $\begin{array}{c}16 \\
(<\mathrm{LOQ}-90)\end{array}$ & $\begin{array}{c}5 \\
(<\mathrm{LOQ}-50)\end{array}$ & $\begin{array}{c}11 \\
(<\mathrm{LOQ}-258)\end{array}$ \\
\hline $2,4^{\prime}-\mathrm{DDE}$ & $<$ LOQ & $\begin{array}{c}1 \\
(<\mathrm{LOQ}-3)\end{array}$ & $\begin{array}{c}7 \\
(<\mathrm{LOQ}-50)\end{array}$ & $\begin{array}{c}34 \\
(<\mathrm{LOQ}-245)\end{array}$ & $\begin{array}{c}4 \\
(<\mathrm{LOQ}-20)\end{array}$ & $\begin{array}{c}22 \\
(<\mathrm{LOQ}-200)\end{array}$ & $\begin{array}{c}11 \\
(<\mathrm{LOQ}-245)\end{array}$ \\
\hline $4,4^{\prime}-\mathrm{DDD}$ & $<$ LOQ & $(<$ LOQ -3$)$ & $<$ LOQ & $(<\mathrm{LOQ}-4)$ & $<$ LOQ & $(<\mathrm{LOQ}-6)$ & $(<\mathrm{LOQ}-6)$ \\
\hline$\sum$ DDTS & $\begin{array}{c}5 \\
(<\mathrm{LOQ}-35)\end{array}$ & $\begin{array}{c}139 \\
(<\text { LOQ-1496) }\end{array}$ & $\begin{array}{c}23 \\
(<\mathrm{LOQ}-57)\end{array}$ & $\begin{array}{c}78 \\
(<\mathrm{LOQ}-326)\end{array}$ & $\begin{array}{c}27 \\
(<\mathrm{LOQ}-94)\end{array}$ & $\begin{array}{c}35 \\
(<\mathrm{LOQ}-215)\end{array}$ & $\begin{array}{c}51 \\
(<\mathrm{LOQ}-1496)\end{array}$ \\
\hline Dieldrin & $<\mathrm{LOQ}$ & $<$ LOQ & $\begin{array}{c}8 \\
(<\mathrm{LOQ}-50)\end{array}$ & $\begin{array}{c}49 \\
(<\mathrm{LOQ}-241)\end{array}$ & $\begin{array}{c}14 \\
(<\mathrm{LOQ}-80)\end{array}$ & $\begin{array}{c}22 \\
(<\mathrm{LOQ}-215)\end{array}$ & $\begin{array}{c}17 \\
(<\mathrm{LOQ}-241)\end{array}$ \\
\hline Lindane & $\begin{array}{c}5 \\
(<\mathrm{LOQ}-30)\end{array}$ & $<$ LOQ & $\begin{array}{c}2 \\
(<\mathrm{LOQ}-20)\end{array}$ & $\begin{array}{c}12 \\
(<\mathrm{LOQ}-40)\end{array}$ & $\begin{array}{c}8 \\
(<\mathrm{LOQ}-40)\end{array}$ & $\begin{array}{c}12 \\
(<\mathrm{LOQ}-40)\end{array}$ & $\begin{array}{c}7 \\
(<\mathrm{LOQ}-40)\end{array}$ \\
\hline
\end{tabular}

OCPs: DDT concentration in this study is in line with observation for Karun River (36 ng/L) [44], Lake Parishan (35.5 ng/L) [45] and Lake Tashk (51 ng/L) [46] but lower than Anzali wetland (108.83 ng/L) [47] and Shahid Rajaei Dam (<LOQ to 100 ng/L) [48] in Iran. Compared with OCP levels in other rivers across 
the globe, the mean concentration of DDT was lower than the Hooghly River (838 ng/L) [49], River Nile (210 ng/L) [50], Pearl River Estuary (143 ng/L) [51] and Iberian Peninsula (164.93 ng/L) [52] but were higher than Moscow River (<LOQ) [53], Yongding River (4.65 ng/L) [54], Chenab River (9.07 ng/L) [55], Ravi River (12.15 ng/L) [56,57] and surface water around Bejing $(0.375 \mathrm{ng} / \mathrm{L})[58]$.

Lindane concentration in this study was lower than Karun River (1585 ng/L) [44], Lake Parishan (58 ng/L) [45], Lake Tashk ( $82 \mathrm{ng} / \mathrm{L})$ [46], Anzali wetland (20 ng/L) [47] and Shahid Rajaei Dam (<LOQ to $170 \mathrm{ng} / \mathrm{L}$ ) [48] in Iran. Compared with studies across the world, lindane concentration were observed to be lower than Ibadan streams (100 ng/L) and Lagos Lagoon (182 ng/L) [58-61]; however, the average concentration was higher than River Nile and Moscow River [50,53], Yongding River $(0.16 \mathrm{ng} / \mathrm{L})$ [54], Chenab River (0.85 ng/L) [55], Ravi River (0.73 ng/L), Uruguay River (2.2 ng/L) [56], Hooghly River (26 ng/L) [49], Pearl River Estuary (13.01 ng/L) [51] and Iberian Peninsula (39.23 ng/L) [52].

Aldrin in water samples of all three rivers in this study were $<$ LOQ but elevated aldrin levels have been reported in Chao Phraya River, Thailand (0.09-7.7 ng/L) [62] and Guanting Reservoir of China (0.60-115 ng/L) [63]. Dieldrin levels in the Talar, Babolrood and Haraz Rivers were 1, 5 and $17 \mathrm{ng} / \mathrm{L}$, respectively. The mean concentration in Talar, Babolrood and Haraz Rivers was lower than Ibadan streams (250 ng/L), River Ero Dam (560 ng/L), River Ero Ondo (740 ng/L), River Osse Ondo (2150 ng/L) [62], Owesse River (1120 ng/L) and Opomu Ondo River (1380 ng/L) [62]. However, it was higher than Iberian Peninsula (0.63 ng/L) [52], River Nile in Egypt (<LOQ) [50], Hooghly River in India (0.4 ng/L), [7], Ravi River in Pakistan (1.588 ng/L) [56] and Uruguay River in Argentina (2.7 ng/L) [59]. Higher average concentration and detection frequency of dieldrin compared with aldrin in water samples might be due to rapid degradation of aldrin to dieldrin [63]. Based on this result, it can be concluded that three rivers from northern Iran have old inputs of aldrin [64].

PCBs: Mean levels of $\sum_{6}$ PCBs in the Haraz, Talar and Babolrood Rivers were 53, 59 and $91 \mathrm{ng} / \mathrm{L}$, respectively. PCB levels in the present study were lower than in a previous study conducted in Iran on inland waters of Shahid Rajaei Dam for $\sum_{13}$ PCB (0-950 ng/L) [48]. The mean concentration of PCBs in Haraz and Talar Rivers was in line with the water samples of International Anzali Wetland, North of Iran $\left(\left(\sum_{7} \mathrm{PCB}, 50.99 \mathrm{ng} / \mathrm{L}\right)\right.$ [47]. $\sum_{6} \mathrm{PCBs}$ in rivers of Iran were higher than $\sum_{8} \mathrm{PCBs}$ from Seine River in France $(7 \mathrm{ng} / \mathrm{L})$ [65,66], $\sum_{26}$ PCBs in Delaware River in the USA $(0.42-1.65 \mathrm{ng} / \mathrm{L})$ [67], $\sum_{12} \mathrm{PCBs}$ in a river network in Shanghai $(8.42 \mathrm{ng} / \mathrm{L})$ and Huangpu River (5.84 ng/L) [68], $\sum_{84} \mathrm{PCBs}$ in surface water around Beijing (21.3 ng/L) [58], $\sum_{19}$ PCBs in Hooghly (57-233) and Brahmaputra (39-161) Rivers in India [7], $\sum_{11}$ PCBs in River Nile (0.286-0544 ng/L) [69-72] and $\sum_{209}$ PCBs in Houston Ship Channel in USA $(2.47 \mathrm{ng} / \mathrm{L})$ [68]. However, PCB levels in the present study were lower than $\sum_{21}$ PCBs in Minjiang River Estuary in China (985.2 ng/L) [69] and $\sum_{27}$ PCBs in surface water from Yamuna in India (332 ng/L) [70].

Levels of OCPs and PCBs at all six sampling stations are given in Tables 1-3. The concentrations of DDTs at Stations 1 and 2 located in the upstream of the Babolrood River, the concentrations of dieldrin at Stations 1 and 2 located upstream of the Haraz and the lindane concentration at Station 2 of Haraz River reported levels <LOQ. The other stations in the downstream of this river reported comparably lower range of the detected POPs. Significant differences between the stations were observed in the study $(p<0.05)$. The higher pollutant concentration specifically in the downstream may be associated with major agricultural activities associated with cotton and rice plantations, human population and soil erosion due to deforestation in the upstream of these rivers. In Talar River, PCB concentration at Station 1 was significantly different from the other stations on Babolrood and Haraz Rivers $(p<0.05)$. Higher POP concentrations in the upstream may be associated with soil erosion due to deforestation and disposal associated with various industries (leather, paper, electronics, fabrics, plastic, etc.) located upstream of these rivers and Station 1.

Overall, the POP concentration was much higher in dry season (summer) than wet season (autumn) (Table 4), which may be attributed to higher dilution factor during wet months $[49,71,72]$. 
Table 4. Concentrations POPs of water samples in the dry and wet seasons in Babolrood, Talar and Haraz Rivers. ${ }^{*} p<0.05$.

\begin{tabular}{|c|c|c|c|c|c|c|}
\hline \multirow{2}{*}{$\begin{array}{c}\text { River } \\
\begin{array}{c}\text { Babolrood } \\
\text { River }\end{array}\end{array}$} & \multicolumn{3}{|c|}{ Dry Season (Number Sampler 32) } & \multicolumn{3}{|c|}{ Wet Season (Number Sampler 36) } \\
\hline & Mean S.D. & Minimum & Maximum & Mean S.D. & Minimum & Maximum \\
\hline PCB & $174 \pm 31^{*}$ & 14 & 559 & $17 \pm 3$ & $<$ LOQ & 61 \\
\hline DDT & $46 \pm 9 *$ & 0 & 180 & $26 \pm 8$ & $<\mathrm{LOQ}$ & 257 \\
\hline Dieldrin & $8 \pm 3^{*}$ & 0 & 50 & $2 \pm 1$ & $<\mathrm{LOQ}$ & 40 \\
\hline Lindane & $<$ LOQ & $<$ LOQ & 0 & $<$ LOQ & $<$ LOQ & $<$ LOQ \\
\hline Haraz River & Mean S.D. & Minimum & Maximum & Mean S.D. & Minimum & Maximum \\
\hline PCB & $95 \pm 104 *$ & 8 & 474 & $14 \pm 14$ & $<\mathrm{LOQ}$ & 60 \\
\hline DDT & $89 \pm 270 *$ & $<\mathrm{LOQ}$ & 1496 & $16 \pm 23$ & $<\mathrm{LOQ}$ & 94 \\
\hline Dieldrin & $34 \pm 66^{*}$ & $<\mathrm{LOQ}$ & 241 & $<$ LOQ & $<\mathrm{LOQ}$ & 1 \\
\hline Talar River & Mean S.D. & Minimum & Maximum & Mean S.D. & Minimum & Maximum \\
\hline PCB & $63 \pm 185^{*}$ & $<\mathrm{LOQ}$ & 1026 & $56 \pm 64$ & $<$ LOQ & 215 \\
\hline DDT & $23 \pm 47 *$ & $<\mathrm{LOQ}$ & 260 & $18 \pm 30$ & $<\mathrm{LOQ}$ & 123 \\
\hline Dieldrin & $1 \pm 2$ & $<\mathrm{LOQ}$ & 6 & $<\mathrm{LOQ}$ & $<\mathrm{LOQ}$ & 6 \\
\hline Lindane & $7 \pm 16$ & $<\mathrm{LOQ}$ & 90 & $<\mathrm{LOQ}$ & $<\mathrm{LOQ}$ & 5 \\
\hline
\end{tabular}

The relative importance of industrial and agricultural sources of chlorinated compounds in the environment can be assessed with the ratio of $\sum$ PCBs to $\sum O C P$ s. A higher ratio is an indication that contamination is dominated by industrial sources and not agricultural [72-75]. In this research, the $\sum \mathrm{PCBs} / \sum \mathrm{OCPs}$ fractions in the surface water samples of Talar, Babolrood and Haraz Rivers are 3.12, 2.8 and 1, respectively. The high values indicate the relative dominance of industrial sources around these rivers and highlight the overall importance of industrial POP residues in Talar and Babolrood Rivers. Intensive usage in industrial activities around these two rivers indicate predominant sources for PCBs influenced by industrial pollution in these locations.

In this study, lindane $(99 \% \gamma-\mathrm{HCH})$ was not detected in Babolrood River but was detected more frequently in Talar (38\%) and Haraz Rivers (22\%) with mean concentrations of 3 and $7 \mathrm{ng} / \mathrm{L}$, respectively. Similar dominance of lindane with major contribution of $\mathrm{HCH}$ isomers was also reported in pesticidal residues of 18 rivers flowing into the southern Caspian Sea. Earlier studies have reported that several chlorinated pesticides, which are part of the Stockholm Convention as POPs, have been detected in the rivers flowing into the Caspian Sea. Among OCPs, the highest concentrations were found for heptachlor (0.005-14.35 ppm) and lindane (at 0.008-40.0 ppm) [74].

The presence of $\gamma-\mathrm{HCH}$ indicates a fresh source of lindane pollution in the studied rivers. Among all $\mathrm{HCH}$ isomers, $\gamma$-HCH has lower reactivity and is the most persistent [75]. This result is in accordance with the findings found in breast milk of mothers near the southern Caspian Sea where previously high levels of lindane were reported [76-78]. Extensive usage of insecticide solutions, such as shampoos containing $1 \%$ lindane, might have resulted in the elevated detection of $\gamma$-HCH in the present study [76].

Biodegradation of DDT into its metabolites in the riverine environment can be a potential source of pesticidal residues. Under aerobic conditions, DDT is biodegraded into DDE, via dehydrochlorination and oxidation. On the other hand, DDT biodegrades into DDD via reductive dichlorination under anaerobic conditions (Syed et al., 2014; Wu et al., 2015a; Oliveira et al., 2016). The (4,4'-DDE+4,4'-DDD)/4,4'-DDT ratio provides an indication of the release of DDTs into the environment [76,78]. A ratio of 0.5 suggests an aged source, while a ratio of $<0.5$ indicates a fresh input. As shown in Figure 2, it was found that $4,4^{\prime}$-DDT and 2,4'-DDT, respectively, were predominant in all three river water samples. On average, $4,4^{\prime}$-DDT collectively accounted for nearly half of total DDTs in the water of Talar and Babolrood Rivers. In Haraz River, $2,4^{\prime}$-DDT contributed $51 \%$ of the total DDT. The $4,4^{\prime}$-DDE $+4,4^{\prime}$-DDD) $/ 4,4^{\prime}$-DDT ratio in water samples of the Talar, Babolrood and Haraz Rivers were 0.13, 0.035 and 0.36, respectively. This ratio and profile of accumulation suggest fresh use of DDT in Iran in this region, and these results are similar to those found for DDT in breast milk and hair samples of mothers and wild cats of Iran, indicating intensive DDT contamination due to illegal usage in Iran $[76,79,80]$. 

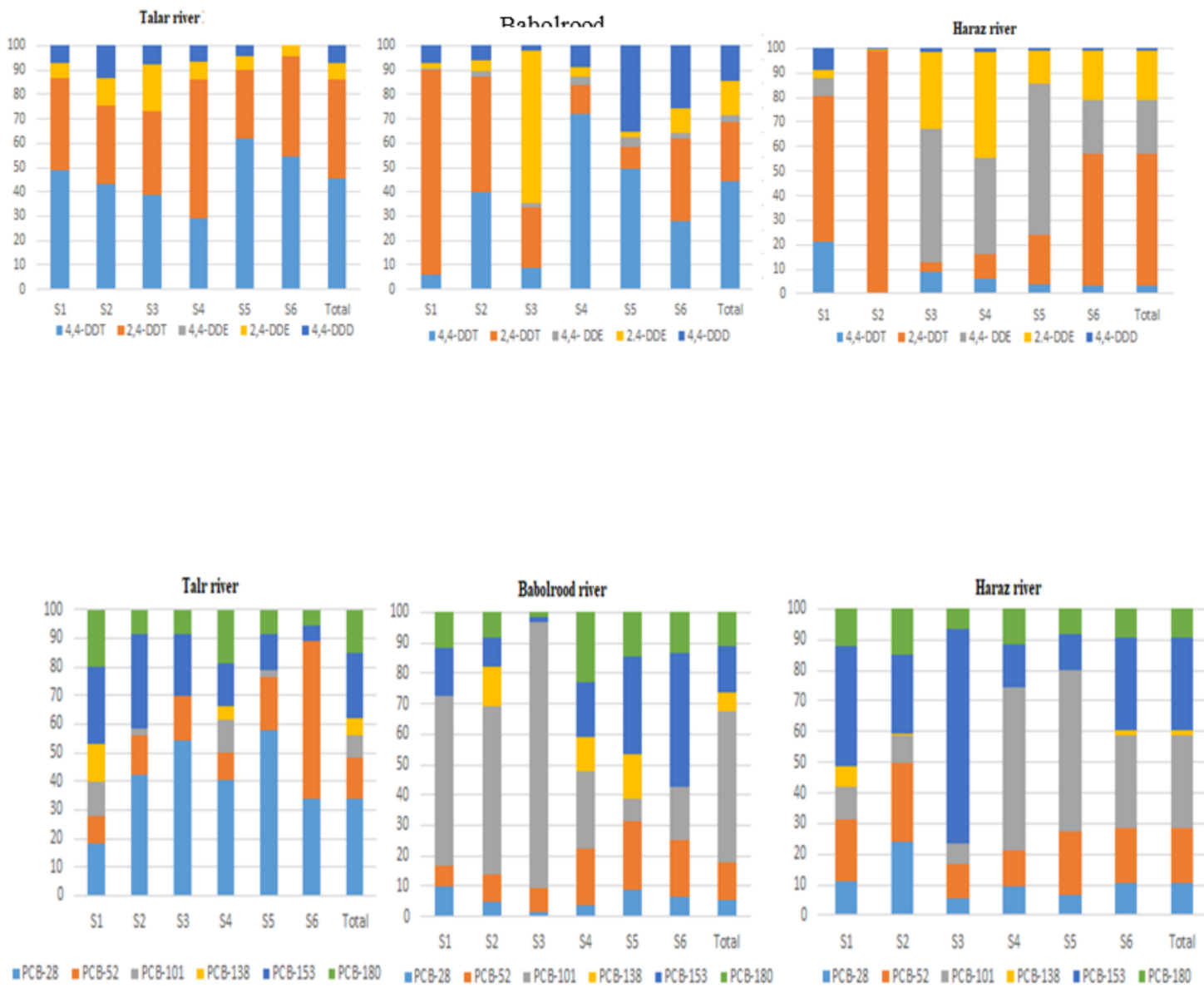

$\|P C B-28 \backsim P C B-52 \backsim P C B-101\| P C B-138 \quad \square P C B-153 \quad \square P C B-180$

$\because P C B 28\|P C B-52 \backsim P C B-101 \quad\| P C B-138 \quad \backsim P C B-153 \quad \backsim P C B-180$

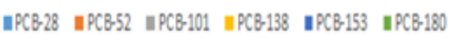

Figure 2. Average percent composition of DDT metabolites and PCB congeners in water samples from different sites (S1-S6) and sum of all sites on the Talar, Babolrood and Haraz Rivers in Iran.

Figure 2 shows the distribution of each $\mathrm{PCB}$ congener in the water samples in these rivers. Dominance of low chlorinated congeners (CB28 and CB52) was observed in Talar River. However, the highly chlorinated congeners (CB101, CB138, CB153 and CB180) were reported at lower levels. Low chlorinated biphenyls (CB28 and CB52) contributed nearly half of the total PCBs in Talar River (Figure 2). Elevated levels of tri- and tetra-chlorinated biphenyls compared to penta- to heptachlor biphenyls in water might be associate to their higher aqueous solubility [81]. Similar compositional profile of PCBs were observed in human milk samples in this region of Iran [78]. On the contrary, predominance of highly chlorinated congeners (CB101 and CB153) and lower concentration of low chlorinated congeners (CB28) was observed in water samples of Babolrood and Haraz Rivers. PCB contamination in water samples of Babolrood and Haraz Rivers reflected industries being a major source [78] with CB101 and CB153 probably associated with the disposal of waste material from industries.

\subsection{Monthly Variations of Legacy POPs}

Talar River: A distinct variation pattern for total PCBs was observed in Talar River and roughly $50 \%$ of total PCBs were observed specifically in July. It is interesting to note that, in October, November and December, there was negligible variations in total PCB concentration between all the stations (Figures 3 and 4). No variation in PCB levels were observed in any of the stations in November. A consistent similar variation in PCBs in all the sampling stations was observed in September. Dominance of PCB levels in July was distinctly observed from Station 1 followed by Station 4 . Similar dominance of PCBs in Station 1 was also observed in August and September. 


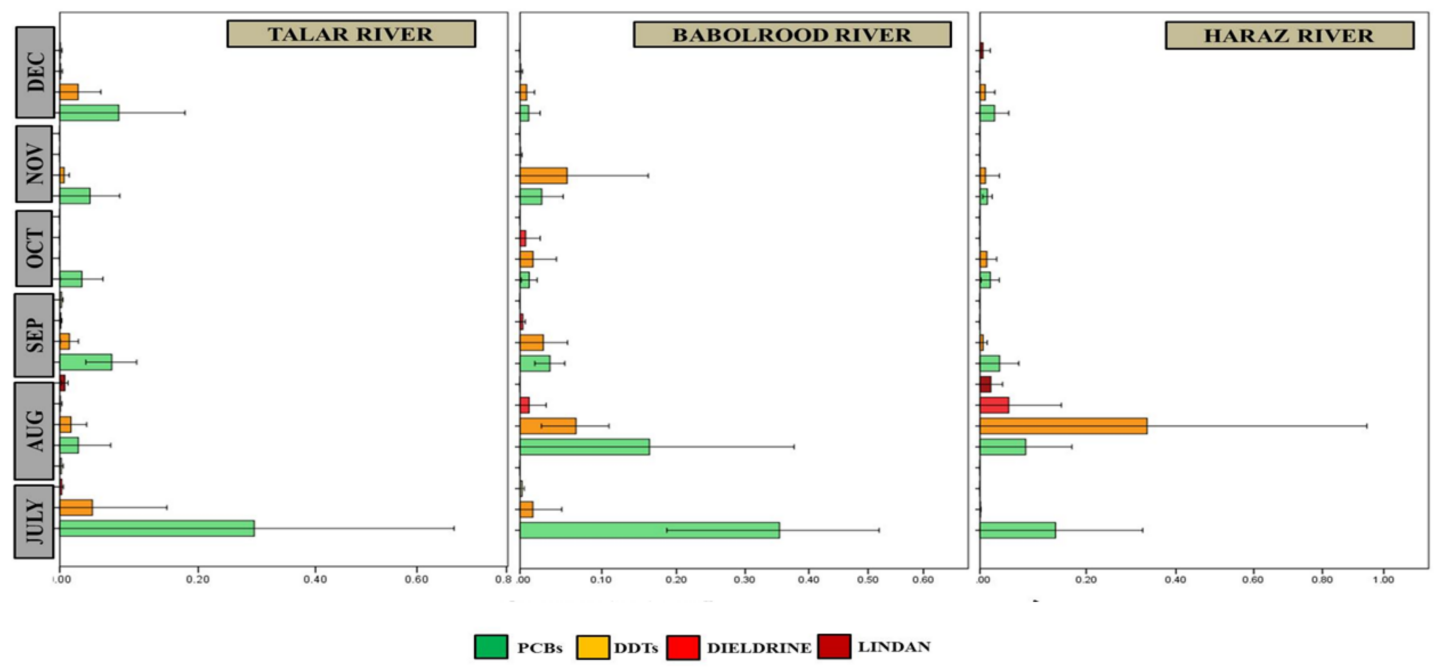

Figure 3. Mean monthly variations of legacy POPs (ng/L) in the Talar, Babolrood and Haraz Rivers at each station.

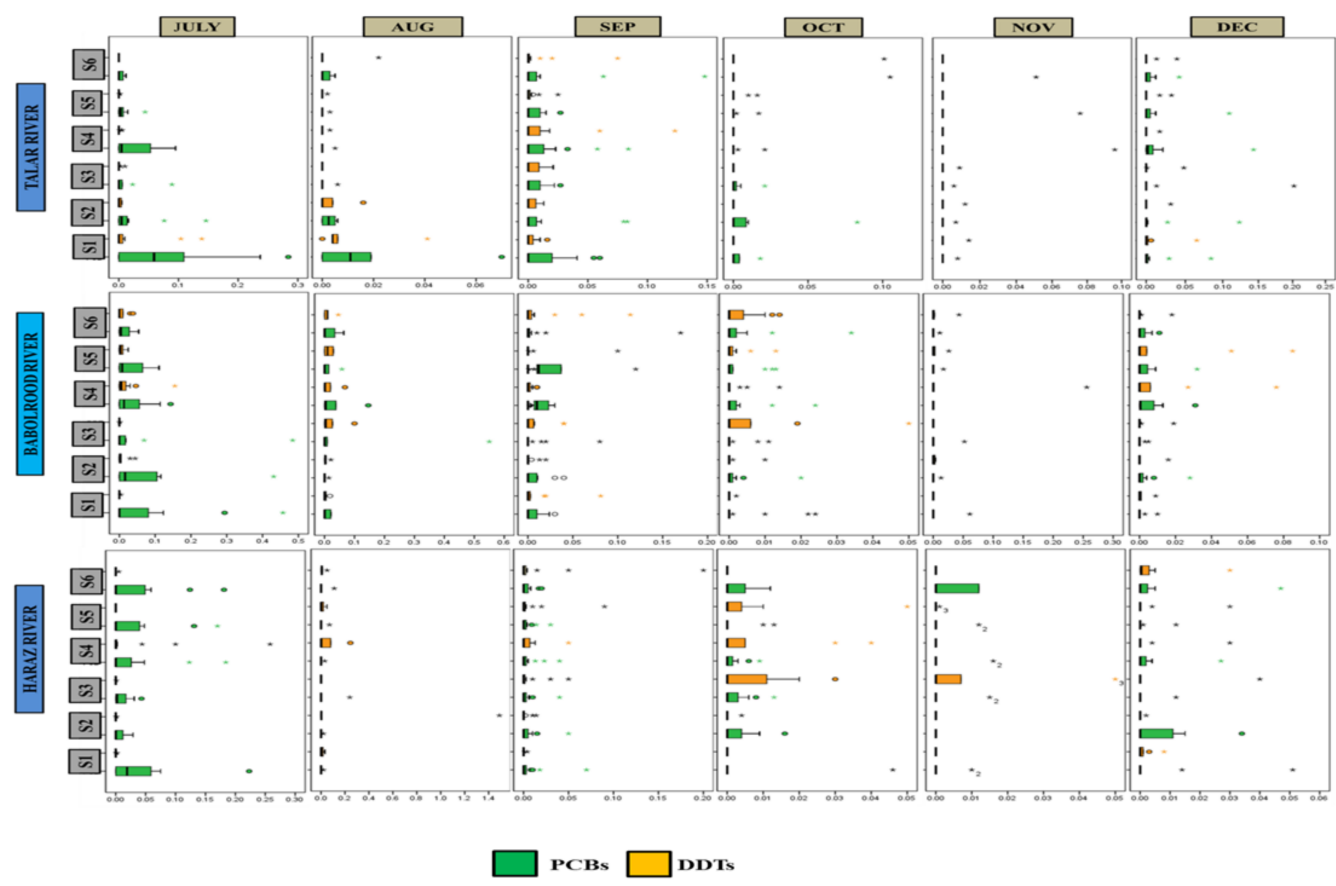

Figure 4. Monthly variations of legacy POPs (PCBs and DDRs) in ng/L for all stations from three rivers of Iran, viz. Talar, Babolrood and Haraz Rivers.

Compared to PCBs, the variations in pesticide were considerably lower in Talar River. Specific dominance of over $75 \%$ of dieldrin and lindane was observed in July and September. Around $40 \%$ of DDT was from September. A similar dominance of DDT was observed in July and December. Similar to PCBs, a consistent trend in variation was observed in September at all sampling stations. No variation in concentration of DDT was observed among any of the sampling stations in October, November and December. Distinct loading of DDT at Stations 1 and 2 was observed in July and August.

The variation of OCPs concentration in the three rivers could be from the differences in the volume and flow of each river in each season related to the impact of rainfall. Other factors such as the land use pattern around the rivers and use of pesticides in specific season might have resulted in the variation of OCP residues in different seasons in these rivers. 
Babolrood River: More than $60 \%$ of the total PCBs in Babolrood River was observed in July, in accordance with the levels observed in Talar River. Similar variation of PCBs consistently at all samplings stations was also observed in July. Specific dominance in PCB concentration was observed at Stations 4 and 5 in September. In addition, a similar trend in variation was also observed in August and October. Further, no variation in PCB levels was observed in November at all sampling stations. The negligible variation in December was similar to the trend in the Talar River.

Among the pesticides, none of the sampling stations in all months detected with lindane. More than $60 \%$ of the total DDT reported in Babolrood River was contributed in July, August and September. More than $90 \%$ of total dieldrin was observed in this river, majorly in July, August and September with the highest level in July. Variation in DDT concentration in September almost predominant at all sampling stations (Figures 3 and 4). No significant variation in DDT levels was observed in October, November and December, excluding a few outlier concentrations. A similar range at Stations 1 and 2 for DDT was also observed in July and August.

Haraz River: The dominance of PCB concentration in July for Haraz River was consistent with Talar and Babolrood Rivers. The variation in PCBs followed a decreasing trend from July to December. Higher loading of PCBs was observed in July and August. Similar variation trends at all sampling stations were observed for July. Specific increment in concentration at Station 6 in October and Station 2 in December was reported. Negligible variation in PCBs at all sampling stations was reported in August and September.

It is interesting to note that roughly $60 \%$ of total DDT reported in Haraz River was from August. The concentration level of DDT followed a decreasing trend from August to December. Dieldrin and lindane were not detected particularly in October and December. However, a moderate increment in levels of dieldrin and lindane was observed in December. Distinct increment in the levels of DDT at Station 4 was observed in August and September. Variation in the level of DDT was evident at most sampling stations in October. A sudden increase of DDT levels was observed at sampling stations in November, possibly associated with its usage for vector control program.

\subsection{Risk Presumption}

The ecotoxicological risk posed by PCBs and OCPs concentrations in water samples from the three rivers in the present study was assessed by comparing the contamination levels with Environmental Quality Standards (EQS) [82]. Surface water concentration of $\sum_{12}$ PCBs levels of Talar, Babolrood and Haraz Rivers were higher than Water Quality Criteria (WQC) $(30 \mathrm{ng} / \mathrm{L})$ and the Criterion Continuous Concentration (CCC) recommended by USEPA (14.0 $\left.\mathrm{ng} \mathrm{L}^{-1}\right)$ [81-83]. The ecotoxicological risk for OCPs was assessed by comparing the levels with European EQS (Directive 2008/105/EC 2008). Further, lindane levels $(\gamma-\mathrm{HCH})$ in surface water of Talar and Haraz Rivers were greater than the ecotoxicological assessment criteria (EAC) $(0.5-5.0 \mathrm{ng} / \mathrm{L})$ [84]. Mean concentrations of DDTs (36-51 $\mathrm{ng} \mathrm{L}^{-1}$ in the Talar, Babolrood and Haraz Rivers) were up to two folds higher than the EQS value of $25.0 \mathrm{ng} \mathrm{L}^{-1}$, thereby indicating high ecological risk due to DDT residues in these rivers. The EQS concentrations of dieldrin in aquatic environments is $30 \mathrm{ng} / \mathrm{L}$. The concentration of this pesticide in the three rivers is lower, showing that the ecological risk is less than EQS. The above assessments indicate that the contamination levels of the studied xenobiotics might pose adverse effects on the river species dwelling in Talar, Babolrood and Haraz Rivers in Iran.

\subsection{Ecotoxicological Concerns}

Ecotoxicological risk for flora and fauna was estimated using the hazard quotient (HQ) approach, as mentioned elsewhere [7]. It was observed that the lower trophic levels in all rivers were generally affected by lindane and DDT. Overall, the risk indicated in summer was considerably higher for both DDT and lindane in all rivers. The risk due to DDT was observed in various trophic levels; however, the risk was observed in one species each in phytoplankton, zooplankton and mollusk groups by lindane in summer. 
Summer season: DDT: Among zooplanktons, scuds and rotifers should be the most affected organisms. HQ value was greater than 1 for scud at more than half of the sites in all rivers (Figure 5). However, the risk was highest in Babolrood River and nearly $80 \%$ of sites showed risk. The risk to rotifer was evident at $19 \%$ of sites in Talar River, $58 \%$ of sites in Babolrood River and $38 \%$ of sites in Haraz River. Dinoflagellate (Amphidinium carterae), diatom (Skeletonema costatum) and phytoplankton were found to be at risk at all sites (wherever DDT was detected) in all the rivers (Figure 5). In mollusk group, only one species, Mytilus edulis, seems to be at risk and high risk was observed in Babolrood River with more than $65 \%$ of sites showing HQ greater than 1 (Figure 5). Among the insect group, mayfly (Baetis sp.) was at risk at nearly half of the sites in Talar and Haraz Rivers and nearly $75 \%$ of sites in Babolrood River. No risk was observed in the fish group (Figure 5).

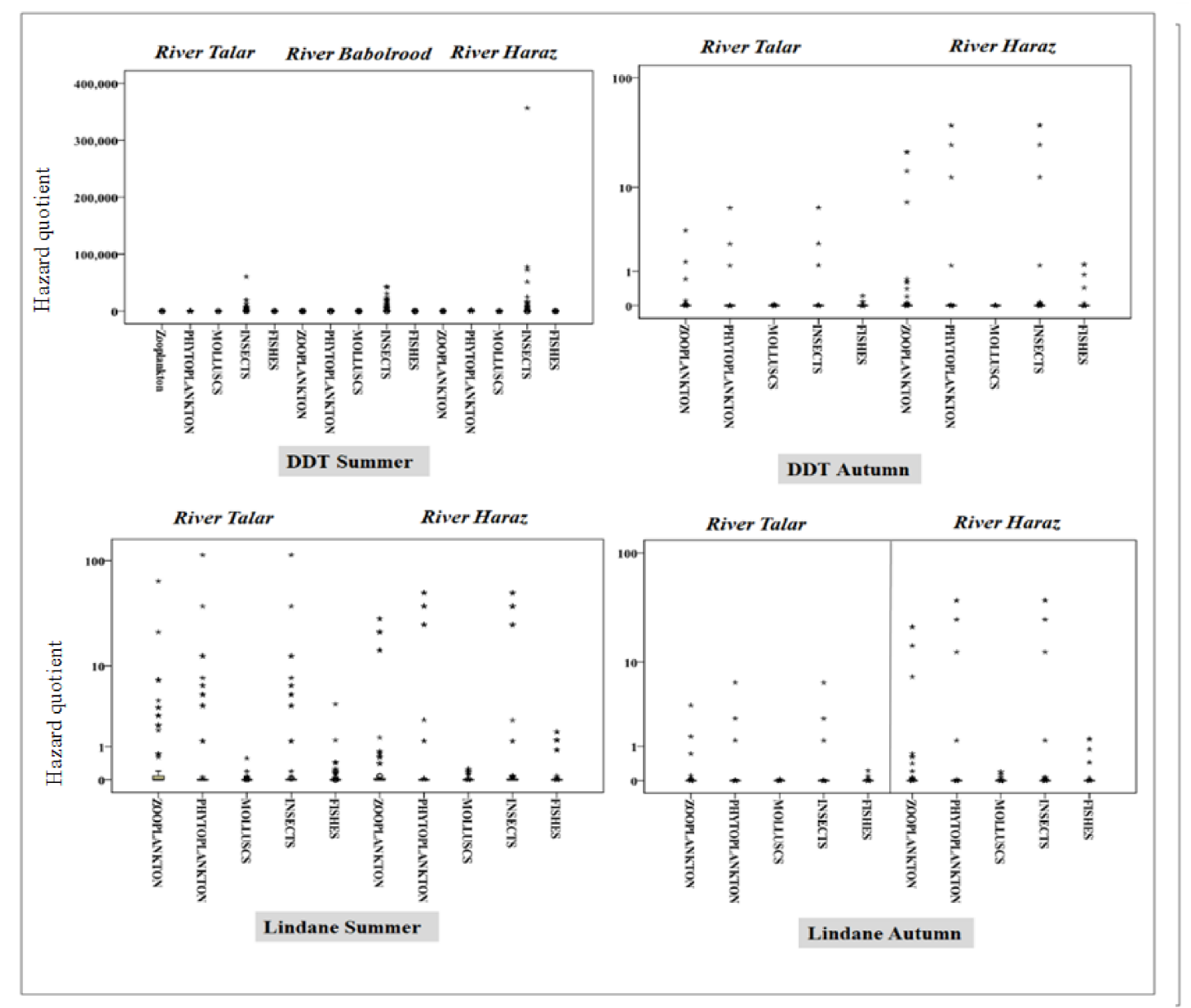

Figure 5. Boxplot showing the range of hazard quotient for organisms in different trophic levels, viz. phytoplankton, zooplankton, insect, mollusk and fish, due to detected concentrations of DDT and lindane in surface water in Talar, Haraz and Babolrood Rivers. Hazard quotient more than 1 indicates ecotoxicological concern to the organism. Hazard quotient = MEC/PNEC (MEC, measured environmental concentration; PNEC, predicted no-effect concentration; see Material and Methods).

Lindane: Water flea (Daphnia magna) showed risk at $63 \%$ of sites in Talar River and all sites where lindane was detected in Haraz River (except two sites) showed risk to water flea. Algae showed risk at all sites in Talar River. Mayfly (Baetis sp.) showed HQ greater than 1 at all sites where lindane was detected in Talar and Haraz Rivers. In the fish group, out of all the sites detected with lindane in Haraz River, more than 50\% showed risk to striped mullet (Mugil cephalus) (Figure 5).

Autumn season: DDT: Risk were low due to DDT in all the rivers; however, in Haraz River, water flea (Daphnia magna), algae, mayfly (Baetis sp.) and striped mullet (Mugil cephalus) indicated risk 
at all sites where DDT was detected. In Talar River, water flea (Daphnia magna), algae (Chlamydonomas reinhardti) and mayfly (Baetis sp.) indicated risk at a few sites but no risk was indicated in Babolrood River.

Lindane: Similar to DDT, lindane showed similar risk on the ecosystem in autumn season. Water flea (Daphnia magna), algae (Chlamydonomas reinhardti), mayfly (Baetis sp.) and striped mullet (Mugil cephalus) were at risk at all sites where lindane was detected. In Talar River, water flea (Daphnia magna), algae (Chlamydonomas reinhardti) and mayfly (Mugil cephalus) indicated risk at a few sites but no risk was observed for organisms in different trophic level in Babolrood River.

\section{Conclusions}

Past usage of OCPs in agricultural sector and selected ongoing applications resulted in OCP contamination in Haraz, Talar and Babolrood Rivers. Apart from illegal use of DDTs, prevalence of other OCPs, particularly lindane in North Province of Iran, might be an indication of continuous influx. Ubiquitous occurrence and elevated levels of OCPs and PCBs in all three rivers might be due to industrial and port activities along the riverbank. We suspect that, in the outer Alborz, industrial activities might influence the release of lighter PCB congeners. All sites had OCP and PCB concentrations above the safety limits for aquatic organisms, indicating probable risk for such organisms. Ecotoxicological risk was higher in summer, particularly for lower trophic level organisms.

Author Contributions: Conceptualization, Formal analysis, data curation, investigation, R.D.B.; methodology, A.E.-S.; review \& editing P.C. All authors have read and agreed to the published version of the manuscript.

Funding: This research received no external funding.

Conflicts of Interest: The authors declare no conflict of interest.

\section{References}

1. Arslan, M.; Imran, A.; Khan, Q.M.; Afzal, M. Plant-bacteria partnerships for the remediation of persistent organic pollutants. Environ. Sci. Pollut. Res. 2017, 24, 4322-4336. [CrossRef] [PubMed]

2. Dai, G.H.; Liu, X.H.; Liang, G.; Xu, M.Z.; Han, X.; Shi, L. Health risk assessment of organochlorine contaminants in fish from a major lake (Baiyangdian lake) in North China. Bull. Environ. Contam. Toxicol. 2011, 87, 58-64. [CrossRef] [PubMed]

3. Sharma, B.M.; Bharat, G.K.; Tayal, S.; Nizzetto, L.; Čupr, P.; Larssen, T. Environment and human exposure to persistent organic pollutants (POPs) in India: A systematic review of recent and historical data. Environ. Int. 2014, 66, 48-64. [CrossRef] [PubMed]

4. Kodavanti, P.R.S.; Loganathan, B.G. Polychlorinated biphenyls, polybrominated biphenyls, and brominated flame retardants. In Biomarkers in Toxicology; Elsevier: Amsterdam, The Netherlands, 2019; pp. 501-518. ISBN 9780128146552.

5. Necibi, M.; Mzoughi, N. The distribution of organic and inorganic pollutants in marine environment. Nov. Sci. Publ. 2017.

6. Strémy, M.; Šutová, Z.; Murínová, L.P.; Richterová, D.; Wimmerová, S.; Čonka, K.; Drobná, B.; Fábelová, L.; Jurečková, D.; Jusko, T.A.; et al. The spatial distribution of congener-specific human PCB concentrations in a PCB-polluted region. Sci. Total Environ. 2019, 651, 2292-2303. [CrossRef] [PubMed]

7. Chakraborty, P.; Khuman, S.N.; Selvaraj, S.; Sampath, S.; Devi, N.L.; Bang, J.J.; Katsoyiannis, A. Polychlorinated biphenyls and organochlorine pesticides in river Brahmaputra from the outer Himalayan Range and river Hooghly emptying into the Bay of Bengal: Occurrence, sources and ecotoxicological risk assessment. Environ. Pollut. 2016, 219, 998-1006. [CrossRef]

8. Eqani, S.A.-M.-A.-S.; Malik, R.N.; Cincinelli, A.; Zhang, G.; Mohammad, A.; Qadir, A.; Rashid, A.; Bokhari, H.; Jones, K.C.; Katsoyiannis, A. Uptake of organochlorine pesticides (OCPs) and polychlorinated biphenyls (PCBs) by river water fish: The case of river Chenab. Sci. Total Environ. 2013, 450-451, 83-91. [CrossRef] [PubMed]

9. Jorjandi, M.A.; Asadikaram, G.; Abolhassani, M.; Fallah, H.; Abdollahdokht, D.; Salimi, F.; Faramarz, S.; Pournamdari, M. Pesticide exposure and related health problems among family members of farmworkers in southeast Iran. A case-control study. Environ. Pollut. 2020, 267, 115424. [CrossRef] [PubMed] 
10. Khuman, S.N.; Chakraborty, P. Air-water exchange of pesticidal persistent organic pollutants in the lower stretch of the transboundary river Ganga, India. Chemosphere 2019, 233, 966-974. [CrossRef]

11. Zhang, G.; Chakraborty, P.; Li, J.; Sampathkumar, P.; Balasubramanian, T.; Kathiresan, K.; Takahashi, S.; Subramanian, A.; Tanabe, S.; Jones, K.C. Passive atmospheric sampling of organochlorine pesticides, polychlorinated biphenyls, and polybrominated diphenyl ethers in urban, rural, and wetland sites along the coastal length of India. Environ. Sci. Technol. 2008, 42, 8218-8223. [CrossRef]

12. Chakraborty, P.; Zhang, G.; Li, J.; Xu, Y.; Liu, X.; Tanabe, S.; Jones, K.C. Selected organochlorine pesticides in the atmosphere of major Indian cities: Levels, regional versus local variations, and sources. Environ. Sci. Technol. 2010, 44, 8038-8043. [CrossRef] [PubMed]

13. Sun, R.; Yu, J.; Liao, Y.; Chen, J.; Wu, Z.; Mai, B. Geographical distribution and risk assessment of dichlorodiphenyltrichloroethane and its metabolites in Perna viridis mussels from the northern coast of the South China Sea. Mar. Pollut. Bull. 2020, 151, 110819. [CrossRef] [PubMed]

14. Dong, W.; Zhang, Y.; Quan, X. Health risk assessment of heavy metals and pesticides: A case study in the main drinking water source in Dalian, China. Chemosphere 2020, 242, 125113. [CrossRef]

15. Ullah, R.; Asghar, R.; Baqar, M.; Mahmood, A.; Alamdar, A.; Qadir, A.; Sohail, M.; Schäfer, R.B.; Eqani, S.A.-M.-A.-S. Assessment of polychlorinated biphenyls (PCBs) in the Himalayan Riverine Network of Azad Jammu and Kashmir. Chemosphere 2020, 240, 124762. [CrossRef] [PubMed]

16. He, W.; Ye, M.; He, H.; Zhu, M.; Li, Y. The decomposition and ecological risk of DDTs and HCHs in the soil-water system of the Meijiang river. Environ. Res. 2020, 180, 108897. [CrossRef]

17. Letcher, R.J.; Bustnes, J.O.; Dietz, R.; Jenssen, B.M.; Jørgensen, E.H.; Sonne, C.; Verreault, J.; Vijayan, M.M.; Gabrielsen, G.W. Exposure and effects assessment of persistent organohalogen contaminants in arctic wildlife and fish. Sci. Total Environ. 2010, 408, 2995-3043. [CrossRef]

18. Baqar, M.; Sadef, Y.; Ahmad, S.R.; Mahmood, A.; Qadir, A.; Aslam, I.; Li, J.; Zhang, G. Occurrence, ecological risk assessment, and spatio-temporal variation of polychlorinated biphenyls (PCBs) in water and sediments along river Ravi and its northern tributaries, Pakistan. Environ. Sci. Pollut. Res. 2017, 24, 27913-27930. [CrossRef]

19. Ali, N.; Malik, R.N.; Mehdi, T.; Eqani, S.A.-M.-A.-S.; Javeed, A.; Neels, H.; Covaci, A. Organohalogenated contaminants (OHCs) in the serum and hair of pet cats and dogs: Biosentinels of indoor pollution. Sci. Total Environ. 2013, 449, 29-36. [CrossRef]

20. Eqani, S.A.-M.-A.-S.; Malik, R.N.; Katsoyiannis, A.; Zhang, G.; Chakraborty, P.; Mohammad, A.; Jones, K.C. Distribution and risk assessment of organochlorine contaminants in surface water from river Chenab, Pakistan. J. Environ. Monit. 2012, 14, 1645-1654. [CrossRef]

21. Arifi, K.; Elblidi, S.; Serghini, A.; Tahri, L.; Yahyaoui, A.; Fekhaoui, M. Taxonomic diversity of benthic macroinvertebrates and bio-evaluation of water quality of Grou river (Morocco) through the use of the standardized global biological index (IBGN). J. Mater. Environ. Sci. 2018, 9, 1343-1355.

22. Gutow, L.; Günther, C.-P.; Ebbe, B.; Schückel, S.; Schuchardt, B.; Dannheim, J.; Darr, A.; Pesch, R. Structure and distribution of a threatened muddy biotope in the south-eastern North Sea. J. Environ. Manage. 2020, 255, 109876. [CrossRef]

23. Majerová, L.; Grygar, T.M.; Elznicová, J.; Strnad, L. The differentiation between point and diffuse industrial pollution of the floodplain of the Ploučnice river, Czech Republic. Water Air Soil Pollut. 2013, 224, 1688. [CrossRef]

24. Tang, W.; Ao, L.; Zhang, H.; Shan, B. Accumulation and risk of heavy metals in relation to agricultural intensification in the river sediments of agricultural regions. Environ. Earth Sci. 2014, 71, 3945-3951. [CrossRef]

25. Ullah, Z.; Khan, H.; Waseem, A.; Mahmood, Q.; Farooq, U. Water quality assessment of the river Kabul at Peshawar, Pakistan: Industrial and urban wastewater impacts. J. Water Chem. Technol. 2013, 35, 170-176. [CrossRef]

26. UNDP. UNDP National Implementation Plan for the Stockholm Convention on Persistent Organic Pollutants; Department for Environment Food \& Rural Affairs: London, UK, 2008.

27. Darvishi, G.; Kootenaei, F.G.; Ramezani, M.; Lotfi, E.; Asgharnia, H. Comparative investigation of river water quality by OWQI, NSFWQI and Wilcox Indexes (case study: The Talar river-Iran). Arch. Environ. Prot. 2016, 42, 41-48. [CrossRef]

28. Mostafalou, S.; Karami-Mohajeri, S.; Abdollahi, M. Environmental and Population studies concerning exposure to pesticides in Iran: A comprehensive review. Iran. Red Crescent Med. J. 2013, 15, 1-8. [CrossRef]

29. Oguchi, T.; Jarvie, H.P.; Neal, C. River water quality in the Humber catchment: An introduction using GIS-based mapping and analysis. Sci. Total Environ. 2000, 251, 9-26. [CrossRef] 
30. Saha, P. Assessment of water quality of Damodar river by water quality index method. Indian Chem. Eng. 2010, 52, 145-154. [CrossRef]

31. Karbassi, A.; Nouri, J.; Ayaz, G. Flocculation of trace metals during mixing of Talar river water with Caspian Seawater. Int. J. Environ. Res. 2007, 1, 66-73.

32. Yousefi, S.; Moradi, H.R.; Keesstra, S.; Pourghasemi, H.R.; Navratil, O.; Hooke, J. Effects of urbanization on river morphology of the Talar river, Mazandarn Province, Iran. Geocarto Int. 2019, 34, 276-292. [CrossRef]

33. Abbasian, H.; Azim, A.; Shamilla, H.; Hamid, G.M. Residues of diazinon in Ab-bandans supplied by Babolroud, Talar and Siaroud rivers, Iran. J. Ecol. Nat. Environ. 2014, 6, 153-158. [CrossRef]

34. Karbassi, A.R.; Nouri, J.; Bidhendi, G.R.N.; Ayaz, G.O. Behavior of $\mathrm{Cu}, \mathrm{Zn}, \mathrm{Pb}, \mathrm{Ni}$ and Mn during mixing of freshwater with the Caspian Sea water. Desalination 2008, 229, 118-124. [CrossRef]

35. Rahmanikhah, Z.; Sari, A.E.; Bahramifar, N.; Bousjien, Z.S. Organophosphorous pesticide residues in surface and ground water in the Southern Coast Watershed of Caspian Sea, Iran. Middle East J. Sci. Res. 2011, 7, 253-259.

36. Mohseni-Bandpei, A.; Yousefi, Z. Status of water quality parameters along Haraz river. Int. J. Environ. Res. 2013, 7, 1029-1038.

37. Nasrabadi, T.; Bidhendi, G.N.; Karbassi, A.; Grathwohl, P.; Mehrdadi, N. Impact of major organophosphate pesticides used in agriculture to surface water and sediment quality (Southern Caspian Sea basin, Haraz river). Environ. Earth Sci. 2011, 63, 873-883. [CrossRef]

38. Farzadkia, M.; Nasseri, S.; Kalantary, R.R.; Asgharnia, H.; Gohari, M.R.; Esrafili, A.; Shahamat, Y.D.; Ghanbari, N. Water quality zoning in babolrood river using national sanitation foundation water quality index and geographic information system. J. Mazandaran Univ. Med. Sci. 2016, 25, 357-362.

39. Pejman, A.H.; Bidhendi, G.R.N.; Karbassi, A.R.; Mehrdadi, N.; Bidhendi, M.E. Evaluation of spatial and seasonal variations in surface water quality using multivariate statistical techniques. Int. J. Environ. Sci. Technol. 2009, 6, 467-476. [CrossRef]

40. Safaripour, M.; Monavari, M.; Zare, M.; Abedi, Z.; Gharagozlou, A. Flood risk assessment using GIS (case study: Golestan Province, Iran). Polish J. Environ. Stud. 2012, 21, 1817-1824.

41. Zhang, Z.L. Environmental Behavior and Primary Assessment of Organic Pesticides in the Estuarine Agricultural Watershed. Ph.D. Thesis, Xiamen University, Xiamen, China, 2001.

42. Reddy, K.N.; Reddy, H. Pesticide residues in surface water of lakes around Hyderabad, India. Pestic. Res. J. 2010, 22, 111-115.

43. Lari, S.Z.; Khan, N.A.; Gandhi, K.N.; Meshram, T.S.; Thacker, N.P. Comparison of pesticide residues in surface water and ground water of agriculture intensive areas. J. Environ. Heal. Sci. Eng. 2014, 12, 11. [CrossRef]

44. Behfar, A.; Nazari, Z.; Rabiee, M.H.; Raeesi, G.; Oveisi, M.R.; Sadeghi, N.; Jannat, B. The organochlorine esticides residue levels in Karun river water. Encycl. Creat. Invent. Innov. Entrep. 2013, 8, 1447. [CrossRef]

45. Kafilzadeh, F.; Shiva, A.; Malekpour, R.; Azad, H. Determination of organochlorine pesticide residues in water, sediments and fish from lake Parishan, Iran. World J. Fish. Mar. Sci. 2012, 4, 150-154. [CrossRef]

46. Kafilzadeh, F. Assessment of organochlorine pesticide residues in water, sediments and fish from lake Tashk, Iran. Achiev. Life Sci. 2015, 9, 107-111. [CrossRef]

47. Javedankherad, I.; Esmaili-Sari, A.; Bahramifar, N. Levels and distribution of organochlorine pesticides and polychlorinated biphenyls in water and sediment from the International Anzali Wetland, North of Iran. Bull. Environ. Contam. Toxicol. 2013, 90, 285-290. [CrossRef] [PubMed]

48. Shakeri, A.; Shakeri, R.; Mehrabi, B. Potentially toxic elements and persistent organic pollutants in water and fish at Shahid Rajaei Dam, north of Iran. Int. J. Environ. Sci. Technol. 2015, 12, 2201-2212. [CrossRef]

49. Mondal, R.; Mukherjee, A.; Biswas, S.; Kole, R.K. GC-MS/MS determination and ecological risk assessment of pesticides in aquatic system: A case study in Hooghly river basin in West Bengal, India. Chemosphere 2018, 206, 217-230. [CrossRef]

50. Dahshan, H.; Megahed, A.M.; Abd-Elall, A.M.M.; Abd-El-Kader, M.A.-G.; Nabawy, E.; Elbana, M.H. Monitoring of pesticides water pollution-The Egyptian river Nile. J. Environ. Heal. Sci. Eng. 2016, 14, 15. [CrossRef] [PubMed]

51. Zhang, Z.; Dai, M.; Hong, H.; Zhou, J.L.; Yu, G. Dissolved insecticides and polychlorinated biphenyls in the Pearl River Estuary and South China Sea. J. Environ. Monit. 2002, 4, 922-928. [CrossRef] 
52. Cruzeiro, C.; Pardal, M.Â.; Rodrigues-Oliveira, N.; Castro, L.F.C.; Rocha, E.; Rocha, M.J. Multi-matrix quantification and risk assessment of pesticides in the longest river of the Iberian peninsula. Sci. Total Environ. 2016, 572, 263-272. [CrossRef]

53. Eremina, N.; Paschke, A.; Mazlova, E.A.; Schüürmann, G. Distribution of polychlorinated biphenyls, phthalic acid esters, polycyclic aromatic hydrocarbons and organochlorine substances in the Moscow river, Russia. Environ. Pollut. 2016, 210, 409-418. [CrossRef]

54. Wang, Y.; Zhang, S.; Cui, W.; Meng, X.; Tang, X. Polycyclic aromatic hydrocarbons and organochlorine pesticides in surface water from the Yongding river basin, China: Seasonal distribution, source apportionment, and potential risk assessment. Sci. Total Environ. 2018, 618, 419-429. [CrossRef] [PubMed]

55. Baqar, M.; Sadef, Y.; Ahmad, S.R.; Mahmood, A.; Li, J.; Zhang, G. Organochlorine pesticides across the tributaries of river Ravi, Pakistan: Human health risk assessment through dermal exposure, ecological risks, source fingerprints and spatio-temporal distribution. Sci. Total Environ. 2018, 618, 291-305. [CrossRef] [PubMed]

56. Shao, Y.; Han, S.; Ouyang, J.; Yang, G.; Liu, W.; Ma, L.; Luo, M.; Xu, D. Organochlorine pesticides and polychlorinated biphenyls in surface water around Beijing. Environ. Sci. Pollut. Res. 2016, 23, 24824-24833. [CrossRef] [PubMed]

57. Unyimadu, J.P.; Osibanjo, O.; Babayemi, J.O. Selected persistent organic pollutants (POPs) in water of river Niger: Occurrence and distribution. Environ. Monit. Assess. 2018, 190. [CrossRef]

58. Mahmood, A.; Malik, R.N.; Li, J.; Zhang, G. Levels, distribution pattern and ecological risk assessment of organochlorines pesticides (OCPs) in water and sediments from two tributaries of the Chenab river, Pakistan. Ecotoxicology 2014, 23, 1713-1721. [CrossRef] [PubMed]

59. Janiot, L.J.; Sericano, J.L.; Roses, O.E. Chlorinated pesticide occurrence in the Uruguay river (Argentina-Uruguay). Water. Air. Soil Pollut. 1994, 76, 323-331. [CrossRef]

60. Boonyatumanond, R.; Tabucanon, M.S.; Siriwong, C.; Prinyatanakun, P. Distribution of organochlorine pesticides in the Chao Phraya river, Thailand. Environ. Monit. Assess. 1997, 44, 315-325. [CrossRef]

61. Xue, N.; Zhang, D.; Xu, X. Organochlorinated pesticide multiresidues in surface sediments from Beijing Guanting reservoir. Water Res. 2006, 40, 183-194. [CrossRef]

62. Osibanjo, O. Organochlorines in Nigeria and Africa. In Persistent Organic Pollutants; Springer: Berlin/Heidelberg, Germany, 2003; pp. 321-354.

63. Blus, L.J.; Henny, C.J.; Kaisser, T.E.; Grove, R.A. Effects on wildlife from use of endrin in Washington State Orchards [Toxicity]. In Proceedings of the Transactions of the North American Wildife and Natural Resources Conferences; Universidad Nacional Agraria: Lima, Peru, 1983.

64. Cailleaud, K.; Forget-Leray, J.; Souissi, S.; Hilde, D.; LeMenach, K.; Budzinski, H. Seasonal variations of hydrophobic organic contaminant concentrations in the water-column of the Seine Estuary and their transfer to a planktonic species Eurytemora affinis (Calanoida, copepoda). Part 1: PCBs and PAHs. Chemosphere 2007, 70, 270-280. [CrossRef]

65. Rowe, A.A.; Totten, L.A.; Xie, M.; Fikslin, T.J.; Eisenreich, S.J. Air- water exchange of polychlorinated biphenyls in the Delaware river. Environ. Sci. Technol. 2007, 41, 1152-1158. [CrossRef]

66. Wang, X.; Han, J.; Bi, C.; Huang, X.; Jia, J.; Chen, Z. Distribution, sources, and risk assessment of polychlorinated biphenyls in surface waters and sediments of rivers in Shanghai, China. Front. Earth Sci. 2017, 11, 283-296. [CrossRef]

67. Omar, W.A.; Mahmoud, H.M. Risk assessment of polychlorinated biphenyls (PCBs) and trace metals in river Nile up-and downstream of a densely populated area. Environ. Geochem. Health 2017, 39, 125-137. [CrossRef] [PubMed]

68. Howell, N.L.; Suarez, M.P.; Rifai, H.S.; Koenig, L. Concentrations of polychlorinated biphenyls (PCBs) in water, sediment, and aquatic biota in the Houston Ship Channel, Texas. Chemosphere 2008, 70, 593-606. [CrossRef]

69. Zhang, Z.L.; Hong, H.S.; Zhou, J.L.; Huang, J.; Yu, G. Fate and assessment of persistent organic pollutants in water and sediment from Minjiang River Estuary, Southeast China. Chemosphere 2003, 52, 1423-1430. [CrossRef]

70. Kumar, B.; Singh, S.K.; Mishra, M.; Kumar, S.; Sharma, C.S. Assessment of polychlorinated biphenyls and organochlorine pesticides in water samples from the Yamuna river. J. Xenobiotics 2012, 2, e6. [CrossRef]

71. Kaushik, C.P.; Sharma, H.R.; Kaushik, A. Organochlorine pesticide residues in drinking water in the rural areas of Haryana, India. Environ. Monit. Assess. 2012, 184, 103-112. [CrossRef]

72. Sharip, Z.; Hashim, N.; Suratman, S. Occurrence of organochlorine pesticides in a tropical lake basin. Environ. Monit. Assess. 2017, 189, 560. [CrossRef] 
73. De Mora, S.; Villeneuve, J.-P.; Sheikholeslami, M.R.; Cattini, C.; Tolosa, I. Organochlorinated compounds in Caspian Sea sediments. Mar. Pollut. Bull. 2004, 48, 30-43. [CrossRef]

74. Nazari, Z.; Haghghi, S.; Eimandel, K.; Hosseini, S.; Parriz, M.; Kalantari, H. Determination of organochlorine pesticides residue in rivers to Caspian sea and sea stations. In Proceedings of the Second National Conference on Optimum Utilization of Chemical Fertilizers and Pesticides in Agriculture; Ministry of Agricultural: Karaj, Iran, 2001.

75. Wang, H.; He, M.; Lin, C.; Quan, X.; Guo, W.; Yang, Z. Monitoring and assessment of persistent organochlorine residues in sediments from the Daliaohe river Watershed, Northeast of China. Environ. Monit. Assess. 2007, 133, 231-242. [CrossRef]

76. Dahmardeh Behrooz, R.; Sari, A.E.; Bahramifar, N.; Ghasempouri, S.M. Organochlorine pesticide and polychlorinated biphenyl residues in human milk from the Southern Coast of Caspian Sea, Iran. Chemosphere 2009, 74, 931-937. [CrossRef]

77. Mohammed, A.; Peterman, P.; Echols, K.; Feltz, K.; Tegerdine, G.; Manoo, A.; Maraj, D.; Agard, J.; Orazio, C. Polychlorinated biphenyls (PCBs) and organochlorine pesticides (OCPs) in harbor sediments from Sea Lots, Port-of-Spain, Trinidad and Tobago. Mar. Pollut. Bull. 2011, 62, 1324-1332. [CrossRef] [PubMed]

78. Li, W.; Yang, H.; Jiang, X.; Liu, Q.; Sun, Y.; Zhou, J. Residues and distribution of organochlorine pesticides in water and suspended particulate matter from Hangzhou Bay, East China Sea. Bull. Environ. Contam. Toxicol. 2016, 96, 295-302. [CrossRef] [PubMed]

79. Dahmardeh Behrooz, R.; Poma, G.; Covaci, A. Assessment of persistent organic pollutants in hair samples collected from several Iranian wild cat species. Environ. Res. 2020, 183, 109198. [CrossRef]

80. Dahmardeh Behrooz, R.; Barghi, M.; Bahramifar, N.; Esmaili-Sari, A. Organochlorine contaminants in the hair of Iranian pregnant women. Chemosphere 2012, 86, 235-241. [CrossRef]

81. Rissato, S.R.; Galhiane, M.S.; Ximenes, V.F.; de Andrade, R.M.B.; Talamoni, J.L.B.; Libânio, M.; de Almeida, M.V.; Apon, B.M.; Cavalari, A.A. Organochlorine pesticides and polychlorinated biphenyls in soil and water samples in the Northeastern part of Sao Paulo State, Brazil. Chemosphere 2006, 65, 1949-1958. [CrossRef]

82. USA EPA. National Recommended Water Quality Criteria; USA Environmental Protection Agency, Office of Water, Office of Science and Technology: Washington, DC, USA, 2010.

83. Lee, K.-T.; Tanabe, S.; Koh, C.-H. Contamination of polychlorinated biphenyls (PCBs) in sediments from Kyeonggi Bay and nearby areas, Korea. Mar. Pollut. Bull. 2001, 42, 273-279. [CrossRef]

84. OSPAR Commission. Quality Status Report 2000; OSPAR Commission: London, UK, 2000; Volume 3.

Publisher's Note: MDPI stays neutral with regard to jurisdictional claims in published maps and institutional affiliations.

(C) 2020 by the authors. Licensee MDPI, Basel, Switzerland. This article is an open access article distributed under the terms and conditions of the Creative Commons Attribution (CC BY) license (http://creativecommons.org/licenses/by/4.0/). 